\title{
Mechanics of Mechanochemically Responsive Elastomers
}

\author{
Qiming Wang ${ }^{1,2}$, Gregory R. Gossweiler ${ }^{3}$, Stephen L. Craig ${ }^{3}$, Xuanhe Zhao ${ }^{1,2, *}$ \\ ${ }^{1}$ Soft Active Materials Laboratory, Department of Mechanical Engineering, Massachusetts Institute of \\ Technology, Cambridge, MA 02139, USA; ${ }^{2}$ Department of Mechanical Engineering and Materials \\ Science, Duke University, Durham, NC 27708, USA. ${ }^{3}$ Department of Chemistry, Duke University, \\ Durham, NC 27708, USA. ${ }^{4}$ Department of Civil and Environmental Engineering, Massachusetts Institute \\ of Technology, Cambridge, MA 02139, USA. \\ *Email: zhaox@mit.edu
}

\begin{abstract}
Mechanochemically responsive (MCR) polymers have been synthesized by incorporating mechanophores - molecules whose chemical reactions are triggered by mechanical force - into conventional polymer networks. Deformation of the MCR polymers applies force on the mechanophores and triggers their reactions, which manifest as phenomena such as changing colors, varying fluorescence and releasing molecules. While the activation of most existing MCR polymers requires irreversible plastic deformation or fracture of the polymers, we covalently coupled mechanophores into the backbone chains of elastomer networks, achieving MCR elastomers that can be repeatedly activated over multiple cycles of large and reversible deformations. This paper reports a microphysical model of MCR elastomers, which quantitatively captures the interplay between the macroscopic deformation of the MCR elastomers and the reversible activation of mechanophores on polymer chains with non-uniform lengths. Our model consistently predicts both the stress-strain behaviors and the color or fluorescence variation of the MCR elastomers under large deformations. We quantitatively explain that MCR elastomers with timeindependent stress-strain behaviors can present time-dependent variation of color or fluorescence due to the kinetics of mechanophore activation and that MCR elastomers with different chain-length distributions can exhibit similar stress-strain behaviors but very different colors or fluorescence. Implementing the model into ABAQUS subroutine further demonstrates our model's capability in guiding the design of MCR elastomeric devices for applications such as large-strain imaging and color and fluorescence displays.
\end{abstract}


Keywords: Mechanochemistry, Stimuli-responsive polymer, Constitutive model, Interpenetrating model, Eight-chain model. 


\section{Introduction}

Polymers capable of chemical reactions in response to external mechanical stimuli offer great promise for developing future sensors, memories, information displays and photomodulators (Beyer and Clausen-Schaumann, 2005; Caruso et al., 2009; Sagara and Kato, 2009). Recent years, a novel strategy for fabricating mechanochemically responsive (MCR) polymers has been developed by covalently incorporating molecules capable of force-triggered chemical reactions, or so-called mechanophores, into polymer networks (Beyer and Clausen-Schaumann, 2005; Black et al., 2011a; Caruso et al., 2009; Kean and Craig, 2012). Deformation of MCR polymers stretches polymer chains which apply forces on the mechanophores and triggers their reactions, leading to phenomena such as changing colors, varying fluorescence and releasing molecules of the MCR polymers. The working mechanism of MCR polymers motivates material design on three levels (Fig. 1): (1) On the molecular level, various mechanophores are synthesized for force-dependent chemical reactions, giving externally detectable chemical or optical signals (Fig. 1a) (Beyer and Clausen-Schaumann, 2005; Black et al., 2011a; Caruso et al., 2009; Kean and Craig, 2012). (2) On the network level, to control the forces applied on mechanophores, the mechanophore-bearing polymer chains can be crosslinked into different network architectures, upon which the macroscopic deformation will be applied (Fig. 1b) (Beiermann et al., 2014; Kingsbury et al., 2011; Lee et al., 2013). (3) On the structure level, to control the macroscopic deformations, the MCR polymers are tailored into various designed structures, which can be deformed by external stimuli such as applied forces, swelling, electric and magnetic fields (Fig. 1c) (Caruso et al., 2009; Lee et al., 2014; Wang et al., 2014b). Through the three-level designs, the MCR polymers have been assembled into functional MCR devices for applications as diverse as controlled color display, fluorescence emission, release of catalyst and small molecules, and constructive network remodeling (Chen et al., 2012; Davis et al., 2009; Hickenboth et al., 2007; Lenhardt et al., 2010; Ramirez et al., 2013).

During the design of MCR devices, it is highly desirable that the MCR polymers can be reversibly and repeatedly activated and deactivated. However, most existing MCR polymers are in glassy 
or semicrystalline states, whose activation requires irreversible plastic deformation or fracture of the polymers. As a result, these MCR polymers cannot fully recover their initial shapes after the first activation, and therefore cannot be activated reversibly or repeatedly (Black et al., 2011b; Chen et al., 2014; Davis et al., 2009; Ducrot et al., 2014; Lee et al., 2013; Lee et al., 2010; Lenhardt et al., 2011; O'Bryan et al., 2010). While mechanophores have been incorporated into thermoplastic elastomers (Beiermann et al., 2014; Chen et al., 2014; Davis et al., 2009; Larsen and Boydston, 2014; Lee et al., 2010; Zhang et al., 2014), little success has yet been achieved to activate the MCR polymer repeatedly to fully recover its initial shape at the room temperature.

Recently, we developed new MCR elastomers by covalently coupling spiropyrans (Davis et al., 2009) - mechanophores that can change color and emit florescence under sufficiently high forces - into the backbone chains of a highly stretchable elastomer network, polydimethylsiloxane (PDMS) Sylgard 184 (Gossweiler et al., 2014; Wang et al., 2014b). The MCR elastomers can recover their initial shapes under multiple cycles of large deformation, allowing for reversible and repeated variations of the color and fluorescence of the elastomers on demand. The MCR elastomers can also be assembled into devices such as a display that is remotely controlled by external physical stimuli (e.g., electric fields) to generate on-demand fluorescent and color patterns. These MCR elastomer systems open promising venues for creating flexible MCR devices with diverse applications in flexible displays, optoelectronics, biomedical luminescent devices and dynamics camouflage skins (Wang et al., 2014b). Despite the potential, the microscopic mechanical details underlying the performance of the MCR elastomers have yet to be investigated, and those mechanistic insights might enable the optimization of MCR elastomer response. For example, the relationship between the macroscopic deformation of the MCR elastomers, the distribution of polymer chain lengths in the network, and the extent of activation of mechanophores embedded along those polymer chains is not understood. This understanding, if achieved, might help guide the development of MCR elastomers, and improve the design of various MCR-elastomer devices (Fig. 1). Recently, Silberstein et al. developed the first multiscale framework aimed at understanding the 
activation of mechanophores within the glassy matrix and viscous rubbery networks (Silberstein et al., 2014; Silberstein et al., 2013). These models probe activation modes that are coupled to irreversible deformation of MCR polymers, and they do not capture the activation within overstressed but elastic, reversible systems. In addition, they do not account for the effect of polymer-chain-length inhomogeneity on mechanophore activations.

In this paper, we report a microphysical model of MCR elastomers, which can quantitatively characterize the process that the macroscopic deformation of the MCR elastomers reversibly activates microscopic mechanophores on polymer chains with intrinsically non-uniform lengths (Erman and Mark, 1997; Flory, 1953; Mark, 1999; Rubinstein and Colby, 2003; Treloar, 1975). We model the MCR elastomer as an interpenetrating network composed of elastomer chains with different lengths of defined distributions. Therefore, the same macroscopic deformation of the elastomer will induce different microscopic forces applied on the polymer chains with different lengths, and thus activates the mechanophores to change color and fluorescence in a non-uniform manner. We further adopt the Bell-like model (Bell, 1978) to account for the reversible activation of mechanophores coupled on different polymer chains of the MCR elastomer network. As a result, our microphysical model can quantitatively predict both the stress-strain behaviors and color/fluorescence variation of the MCR elastomers under multiple cycles of large deformation. In particular, we quantitatively explained: (1) MCR elastomers with time-independent stress-strain behaviors can present time-dependent variation of color or fluorescence due to the kinetics of mechanophore activation; and (2) MCR elastomers with different chain-length distributions exhibit similar stress-strain behaviors, but very different colors/fluorescence under the same deformation, indicating a critical requirement of including contributions of inhomogeneous chain-length distributions to model activation behavior of chain-bound mechanophores. In addition, we program the new constitutive model into ABAQUS user subroutine (SIMULIA, USA), which is able to predict the activation of MCR-elastomer structures with complicated geometries undergoing inhomogeneous deformations. 
The plan of the paper is as follows. Section 2 introduces the experimental observation of the fluorescence emission of the uniaxially stretched MCR elastomers. Section 3 presents a microphysical model which integrates an interpenetrating-network model with non-uniform chain lengths and the Belllike model for activation of mechanophores. Thereafter, the constitutive models are discussed in Section 4 by considering various chain-length distributions. Focusing on the log-normal chain-length distribution, we develop an ABAQUS UHYPER subroutine, with which in section 5 we show the numerical simulations match well with experimental observations of the fluorescence emission of the MCR elastomers in the applications of strain imaging and fluorescence displays. The concluding remarks are given in Section 6.

\section{Experimental}

The MCR elastomer is fabricated by dissolving a bis-alkene functionalized spiropyran solution into the poly(dimethyl siloxane) (PDMS) Sylgard 184 pre-polymer. During the polymerization, the spiropyrans are crosslinked into the Sylgard network by a platinum catalyzed hydrosilylation reaction, the same curing reaction as that between vinyl- and hydrosilane functionalities in the initial Sylgard networks (Fig. 2). Normal vinyl Sylgard chains and spiropyran bearing vinyl Sylgard chains coexist in the polymer network (Fig. 2). Details of the fabrication can be found elsewhere (Gossweiler et al., 2014; Wang et al., 2014b). The thickness of the MCR film is controlled by the spin-coat speed of pre-polymer cast onto BoPET film (McMaster-Carr, USA). The stress-strain relations of the MCR elastomer are measured by the uniaxial tension tests (Micro-strain analyzer, TA instruments, USA).

The MCR elastomer under large deformation (i.e., >125\% strain) applies sufficiently high forces to the spiropyran mechanophores to trigger a 6- $\pi$ electrocyclic ring-opening reaction that reversibly transforms a near-colorless spiropyran to a highly-colored and fluorescent merocyanine (Davis et al., 2009; Lee et al., 2013; Lee et al., 2010) (Fig. 2). The transformation from spiropyran to merocyanine can 
also be driven by the exposure of ultraviolet light (UV, $\sim 365 \mathrm{~nm}$ ) or heat. The reverse reaction (from mecrocyanine to spiropyran) can be accelerated by relaxing the applied force and exposing to visible light (e.g., green light, wavelength $\sim 545 \mathrm{~nm}$ ). In a typical test of fluorescent/color variation, an MCR film $(\sim 300 \mu \mathrm{m})$ is first driven to a photostationary equilibrium by illuminating a green light (wavelength $\sim 545$ $\mathrm{nm}$ ) for $3 \mathrm{~min}$, and then stretched uniaxially or equibiaxially. In uniaxial tests, the MCR films are stretched under a pure-shear condition with initial gauge length $\sim 6 \mathrm{~mm}$ and width $\sim 20 \mathrm{~mm}$ (Fig. 3a). The gauge length for the equibiaxial tests is $\sim 10 \mathrm{~mm}$ by $\sim 10 \mathrm{~mm}$. The deformation of the MCR films is

measured by stretches (Fig. 3b) which are defined as $\lambda=L / L_{0}$ where $L_{0}$ and $L$ are the gauge lengths of the elastomer film in the undeformed and deformed states, respectively (Fig. 3a). During the fluorescent/color tests, the MCR film is held at each deformed state for sufficiently long time that allows the activation of mechanophores to reach equilibriums. As shown in Fig. 3a, deformation of the MCR film indeed varies its color from the initially pale yellow to dark blue. In addition, the MCR film is imaged by a fluorescent microscope (Nikon Eclipse LV100) with a Nikon Intensilight C-HGFI mercury lamp as the illumination source. The objective is a 5x MUE41050 LU Plan Fluor. The fluorescent filter is TRITC Hy Q Cube (EX530-560, DM570, BA590-650). Images are acquired on the film center with a Nikon DS-Qi1Mc camera and saved as a 16-bit grayscale tiff images (width $2.22 \mathrm{~mm}$ and $1.78 \mathrm{~mm}$ height). For comparison, fluorescent images have been colored using the LUT command (Red Hot) of Image J. Since the film is relatively thin $(\leq 400 \mu \mathrm{m})$, we assume the observed fluorescence indicates the number of merocyanines throughout the thickness of the film (see Eq. 36). As shown in Fig. 3b, the MCR elastomers exhibit different fluorescent intensities under different degree of stretches.

We further quantify the numerical value of fluorescent intensity $Q$ by integrating the gray pixel intensity in the acquired image, and plot the intensity as a function of stretches in the film $\lambda$ under uniaxial or equibiaxial tensions. We denote the fluorescent intensity of the film at undeformed state as $Q_{0}$. As shown on Fig. 3c, the curves of normalized fluorescent intensity $Q / Q_{0}$ vs. stretch $\lambda$ for 
MCR films with different concentrations of mechanophores coincide with one another. In addition, the fluorescence intensity first decreases slightly at a low stretch ratio (i.e., $\lambda<2$ for uniaxial and $\lambda<1.6$ for equibiaxial), and then increases monotonically with deformation of the MCR film. This trend of slight decrease to drastic increase can be understood as follows. Under low stretches, only a small portion of the mechanophores in the MCR film are activated, mostly on relatively short chains; but the observed quantity of mechanophores throughout the film also decreases as the MCR film is stretched thinner. Therefore, the observed fluorescence intensity slightly decreases with the increasing stretch (Fig. 3c). However, as the stretch becomes large (i.e., $\lambda<2$ for uniaxial and $\lambda<1.6$ for equibiaxial), a significant portion of mechanophores are activated. Despite the offset of the reduced film thickness, the overall fluorescence intensity still increases with the stretch in the MCR film (Fig. 3c).

\section{The theoretical model}

\subsection{Interpenetrating networks with non-uniform chain lengths}

\subsubsection{The interpenetrating network model}

Polymer chains in most polymer networks have non-uniform lengths, owing to the stochastic nature of polymerization and/or crosslinking of the networks (Erman and Mark, 1997; Flory, 1953; Mark, 1999; Rubinstein and Colby, 2003; Treloar, 1975). In particular, the lengths of polymer chains within the PDMS used for the current MCR elastomers indeed distribute across a wide range (Erman and Mark, 1997; Mark, 1999). Existing network-based constitutive models for elastomers usually assume a uniform chain-length distribution in polymer networks, since these models are primarily focused on overall stressstrain behaviors of the elastomers (Flory, 1953; Rubinstein and Colby, 2003; Treloar, 1975). However, the activation of mechanophores within the MCR elastomers is expected to be sensitive to chain-length distribution. When the elastomer network is under macroscopic deformation, the forces in polymer chains with inhomogeneous lengths should be significantly distinctive. The inhomogeneous chain forces in turn 
activate the mechanophores in dramatically different levels. Here we will develop a simple network model that accounts for the non-uniform chain-length within the MCR elastomer.

In the current paper, we target at developing a general model that can account for the essential feature and effects of non-uniform distribution of polymer-chain lengths in MCR elastomers, without the complication of detailed microstructures of the elastomers (e.g., reinforcement of nanoparticles in PDMS). To achieve this goal, we model the elastomer network as being composed of multiple crosslinked polymer networks interlaced with one another on a molecular scale, where each network spans the whole volume of the elastomer (Zhao, 2012) (Fig. 4).

A polymer chain on a network is defined as the segment of molecules between two successive chemical crosslinks (Flory, 1953; Rubinstein and Colby, 2003; Treloar, 1975), and the chain can be effectively divided into a number of freely-joint rigid links, each link with the same length $l$, so-called Kuhn length (Kuhn and Grün, 1942). We assume that the MCR elastomer consists of $m$ types of interpenetrated networks and that the link number of polymer chains in the same type of network is the same (Fig. 4). We denote the number of links on a chain in the network of the ith type (referred as the ith network) as $n_{i}$ (Fig. 4), and the number of polymer chains in the $i$ th network per unit volume of the elastomer as $N_{i}$, where $1 \leq i \leq m$. With respect to the network chains, mechanophore spiropyrans are assumed to be of negligible length with very low concentration (i.e., $\leq 0.5 \% \mathrm{wt}$ ) and homogeneously distributed in the networks, we assume that the presence of spiropyrans on a polymer chain does not affect its number of links. Without loss of generality, we further assume $n_{1} \leq n_{2} \leq \cdots n_{i} \cdots \leq n_{m}$ (Fig. 4b). Therefore, the number of total polymer chains per unit volume of the elastomer can be expressed as

$$
N=\sum_{i=1}^{m} N_{i}
$$


Multiple networks of the same type may coexist in an elastomer to adjust the chain density of this type of network. We assume that the chain density of different types of networks follows a statistical distribution as

$$
\frac{N_{i}}{N}=p_{i}\left(n_{i}\right)
$$

where the summation of the probability densities is equal to a unit, i.e., $\sum_{i=1}^{m} p_{i}\left(n_{i}\right)=1$.

Assuming all networks in the MCR elastomer are incompressible, the volume of the ith network per unit volume of the elastomer can be expressed as

$$
V_{i}=\frac{N_{i} n_{i} v_{i}}{\sum_{j=1}^{m} N_{j} n_{j} v_{j}}
$$

where $v_{i}$ is the volume of a link on polymer chains in the ith network. Since the links on all networks are the same in elastomers used in the current study, we have

$$
V_{i}=\frac{N_{i} n_{i}}{\sum_{j=1}^{m} N_{j} n_{j}}
$$

\subsubsection{Microscopic force on polymer chains in the ith network}

The fully-extended contour length of a polymer chain on the $i$ th networks can be expressed as $r_{i}^{c}=n_{i} l$. At the undeformed state of the elastomer, the end-to-end distance of a chain on the $i$ th network can be approximated as $r_{i}^{0}=\sqrt{n_{i}} l$ (Rubinstein and Colby, 2003; Treloar, 1975). Under deformation, the end-to-end distance of the chain will change to $r_{i}$, and the stretch of the chain is defined as 


$$
\Lambda_{i}=\frac{r_{i}}{r_{i}^{0}}
$$

where chain stretch must be in a range, $1 \leq \Lambda_{i} \leq r_{i}^{c} / r_{i}^{0}=\sqrt{n_{i}}$.

According to Langevin-chain statistics (Kuhn and Grün, 1942), the free energy of a deformed chain on ith network can be expressed as

$$
w_{i}=n_{i} k_{B} T\left(\frac{\beta_{i}}{\tanh \beta_{i}}+\ln \frac{\beta_{i}}{\sinh \beta_{i}}\right)
$$

where $\beta_{i}=L^{-1}\left(\Lambda_{i} / \sqrt{n_{i}}\right), L(x)$ is Langevin function defined as $L(x)=\operatorname{coth}(x)-1 / x, L^{-1}(x)$ is the inverse of the Lagevin function, $k_{B}=1.38 \times 10^{-23} \mathrm{~m}^{2} \mathrm{~kg} \cdot \mathrm{s}^{-2} \mathrm{~K}^{-1}$ is the Boltzmann constant, and $T$ is the absolute temperature in Kelvin. Here, we assume the presence of mechanophores does not affect the free energy of stretching the polymer chain. The force in the deformed a chain on the ith network can be derived as (Treloar, 1975)

$$
f_{i}=\frac{\partial w_{i}}{\partial r_{i}}=\frac{1}{\sqrt{n_{i}} l} \frac{\partial w_{i}}{\partial \Lambda_{i}}=\frac{k_{B} T}{l} L^{-1}\left(\frac{\Lambda_{i}}{\sqrt{n_{i}}}\right)
$$

This chain force $f_{i}$ will be applied on mechanophores coupled on the polymer chains (Fig. $\mathbf{4 b}$ ), and thus affect their chemical reactions. From Eq. 7, we can see that, even under the same stretch (i.e., the same $\Lambda_{i}$ ), polymer chains with different chain lengths (i.e., different $n_{i}$ ) will experience different chain forces. Consequently, the activation of the chain-bound mechanophores will significantly depend on the lengths of polymer chains.

It should be noted that the framework of the current theory is not restricted to the use of Langevin chain model given by Eq. 6, it can readily adopt other models, such as the Worm-Like-Chain 
model as given in the Appendix A (Bischoff et al., 2002a; Bischoff et al., 2002b; Kuhl et al., 2005; Ogden et al., 2006).

\subsubsection{Macroscopic stresses in the MCR elastomer}

In the ith networks, polymer chains can assemble into different structures such as three-chain structure (James and Guth, 1943), four-chain structure (Flory, 1944), eight-chain structure (Arruda and Boyce, 1993) and n-chain structure (Treloar and Riding, 1979; Wu and Van der Giessen, 1992). For simplicity of the analysis, we assume the polymer chains in each network are respectively assembled into cubic elements by following the eight-chain model (Arruda and Boyce, 1993). The cubic elements of different networks homogeneously overlap with one another to form the interpenetrating network (Fig. 4).

When the MCR elastomer is subjected to a macroscopic deformation denoted by principal stretches in three directions, $\lambda_{1}, \lambda_{2}$ and $\lambda_{3}$, the eight-chain cubic elements in each network follow the affine deformation with the same principal stretches of $\lambda_{1}, \lambda_{2}$ and $\lambda_{3}$. Therefore, the stretch of all chains on the ith network can be expressed as (Arruda and Boyce, 1993)

$$
\Lambda_{i}=\sqrt{\frac{I_{1}}{3}}
$$

where $I_{1}=\lambda_{1}^{2}+\lambda_{2}^{2}+\lambda_{3}^{2}$ is the first invariant of the Cauchy Green tensor.

From Eq. 6, the free energy associated with the stretching of the polymer chains in the ith network per unit volume of the MCR elastomer can be calculated as

$$
W_{i}=N_{i} n_{i} k_{B} T\left(\frac{\beta_{i}}{\tanh \beta_{i}}+\ln \frac{\beta_{i}}{\sinh \beta_{i}}\right)
$$


where $\beta_{i}=L^{-1}\left(\Lambda_{i} / \sqrt{n_{i}}\right)$ and $\Lambda_{i}$ is given by Eq. 8. It is noted that the double index in Eq. 9 and other equations in this paper do not follow the Einstein summation convention. Therefore, the total free energy of deforming a unit volume of the MCR elastomer can be expressed as

$$
W=\sum_{i=1}^{m} W_{i}
$$

To account for the incompressibility of the MCR elastomer $\left(\lambda_{1} \lambda_{2} \lambda_{3}=1\right)$, we modify the free energy function in Eq. 10 as

$$
\hat{W}=W-\Pi\left(\lambda_{1} \lambda_{2} \lambda_{3}-1\right)
$$

where $\Pi$ is a Lagrange multiplier used to enforce the incompressibility and can be determined by boundary conditions. Therefore, the nominal principal stresses can be calculated as

$$
\begin{gathered}
s_{1}=\frac{\partial \hat{W}}{\partial \lambda_{1}}=\sum_{i=1}^{m} \frac{\partial W_{i}}{\partial \lambda_{1}}-\Pi \lambda_{2} \lambda_{3} \\
s_{2}=\frac{\partial \hat{W}}{\partial \lambda_{2}}=\sum_{i=1}^{m} \frac{\partial W_{i}}{\partial \lambda_{2}}-\Pi \lambda_{1} \lambda_{3} \\
s_{3}=\frac{\partial \hat{W}}{\partial \lambda_{3}}=\sum_{i=1}^{m} \frac{\partial W_{i}}{\partial \lambda_{3}}-\Pi \lambda_{1} \lambda_{2}
\end{gathered}
$$

And the principal Cauchy stresses can calculated as

$$
\begin{gathered}
\sigma_{1}=\lambda_{1} s_{1}=\sum_{i=1}^{m} \lambda_{1}^{2} \frac{N_{i} \sqrt{n_{i}} k_{B} T \beta_{i}}{3 \Lambda_{i}}-\Pi \\
\sigma_{2}=\lambda_{2} s_{2}=\sum_{i=1}^{m} \lambda_{2}^{2} \frac{N_{i} \sqrt{n_{i}} k_{B} T \beta_{i}}{3 \Lambda_{i}}-\Pi
\end{gathered}
$$




$$
\sigma_{3}=\lambda_{3} s_{3}=\sum_{i=1}^{m} \lambda_{3}^{2} \frac{N_{i} \sqrt{n_{i}} k_{B} T \beta_{i}}{3 \Lambda_{i}}-\Pi
$$

If we consider stretching an MCR elastomer film under the pure-shear condition as illustrated in Fig. 2a, three principal stretches can be expressed as $\lambda_{1}=\lambda, \lambda_{2}=1$ and $\lambda_{3}=1 / \lambda$. By setting $\sigma_{3}=0$, we can obtain the Cauchy stress along the stretched direction in the elastomer film as

$$
\sigma_{1}=\left(\lambda^{2}-\frac{1}{\lambda^{2}}\right) \sum_{i=1}^{m} \frac{N_{i} \sqrt{n_{i}} k_{B} T \beta_{i}}{3 \Lambda_{i}}
$$

The nominal stress $S_{1}$ along the stretch direction can be derived by considering $S_{1}=\sigma_{1} / \lambda$, i.e.,

$$
s_{1}=\left(\lambda-\frac{1}{\lambda^{3}}\right) \sum_{i=1}^{m} \frac{N_{i} \sqrt{n_{i}} k_{B} T \beta_{i}}{3 \Lambda_{i}}
$$

where $\beta_{i}=L^{-1}\left(\Lambda_{i} / \sqrt{n_{i}}\right), \Lambda_{i}=\sqrt{\left(\lambda^{2}+\lambda^{-2}+1\right) / 3}$, and the relation of $N_{i}$ and $n_{i}$ follows Eq. 2 .

\subsection{Force-dependent chemical reaction of mechanophores}

\subsubsection{Kinetics for chemical reaction of mechanophores}

We denote the number of mechanophores per unit volume of the MCR elastomer as $c$. Since the MCR elastomer is incompressible, $c$ is a constant throughout the deformation of the MCR elastomer. Since mechanophores are homogeneously distributed within the MCR elastomer, the number of mechanophores coupled on the ith network is proportional to the volume ratio of the ith network in the elastomer. Therefore, the number of mechanophores on the ith network in a unit volume of the MCR elastomer can be expressed as

$$
c_{i}=c V_{i}
$$


where the volume ratio of the ith network $V_{i}$ is given by Eq. 4 .

Spiropyrans and merocyanines are able to interconvert through a reversible electrocyclic reaction (Fig. 2), which is significantly affected by microscopic forces applied on the mechanophores. It is noted that merocyanines may have two isomers; for simplicity, here we study the reactions of the both merocyanine isomers together (Davis et al., 2009; Silberstein et al., 2014; Silberstein et al., 2013). We denote $c_{i}^{S}$ and $c_{i}^{M}$ as the numbers of spiropyrans and merocyanines on the ith network per unit volume of the MCR elastomer at the current state, respectively. Since the ith network is incompressible, the number of mechanophores in the ith network is a constant throughout the deformation of the MCR elastomer, and $c_{i}=c_{i}^{S}+c_{i}^{M}$. Following the convention in mechanochemistry, we define the conversion from spiropyrans to merocyanines as the forward reaction, and from merocyanines to spiropyrans as the reverse reaction (Fig. 2). The chemical kinetics between spiropyrans and merocyanines on the ith network of the MCR elastomer can be expressed as (Hänggi et al., 1990)

$$
\frac{d c_{i}^{M}}{d t}=k_{i}^{f} c_{i}^{S}-k_{i}^{r} c_{i}^{M}
$$

where $t$ denotes time, $k_{i}^{f}$ is the forward reaction rate (with dimension of $\mathrm{s}^{-1}$ ), and $k_{i}^{r}$ is the reverse reaction rate (with dimension of $\mathrm{s}^{-1}$ ) of mechanophores on the ith network. Since the change of color and fluorescence of the elastomer depends on merocyanine, we will focus on concentrations of merocyanines in MCR elastomers in the current study. Considering $c_{i}=c_{i}^{S}+c_{i}^{M}$, we can calculate the time-dependent number of merocyanines on the ith network per unit volume of the MCR elastomer at the current state $c_{i}^{M}(t)$ by solving an ordinary differential equation,

$$
\frac{d c_{i}^{M}}{d t}=-\left(k_{i}^{f}+k_{i}^{r}\right) c_{i}^{M}+k_{i}^{f} c_{i}
$$


The solution for Eq. 18 is

$$
c_{i}^{M}(t)=\exp \left[-\int_{t_{0}}^{t}\left(k_{i}^{f}+k_{i}^{r}\right) d \tau\right]\left\{\int_{t_{0}}^{t} \frac{k_{i}^{f} c_{i}}{\exp \left[-\int_{t_{0}}^{\tau}\left(k_{i}^{f}+k_{i}^{r}\right) d \xi\right]} d \tau+\left.c_{i}^{M}\right|_{t=t_{0}}\right\}
$$

where $\left.c_{i}^{M}\right|_{t=t_{0}}$ is the concentration of merocyanines on the ith network at an initial time $t_{0}$. The total number of merocyanines in a unit volume of the MCR elastomer at the current state can thus be calculated as

$$
c^{M}(t)=\sum_{i=1}^{m} c_{i}^{M}(t)
$$

From Eq. 19 and Eq. 20, it is evident that the merocyanine concentration at the current state depends on the initial condition $\left.c_{i}^{M}\right|_{t=t_{0}}$, forward and reverse reaction rates $k_{i}^{f}$ and $k_{i}^{r}$, and the current time $t$. We will discuss these dependences in the following sections.

\subsubsection{Force-dependent reaction rates}

The reversible chemical reaction between spiropyrans and merocyanines can be understood by considering the potential energy of the mechanophores during the reaction (Fig. 5) (Kauzmann and Eyring, 1940; Kramers, 1940). The basic assumption is that the dynamics of the chemical reaction can be projected onto a reaction coordinate $x$ (Kauzmann and Eyring, 1940). The potential energy of tworeactant states (spriropyran and merocyanine) and internal transition state can be plotted as a function of the reaction coordinate $x$. The transition between the spiropyrans and merocyanines needs to overcome the energy barriers, which are defined as the potential energy difference between the respective reactant states and the transition state (Fig. 5). 
When the force applied to the mechanophore is zero, the potential-energy landscape of the mechanophores is illustrated in Fig. 5a, where $\Delta G^{S}$ and $\Delta G^{M}$ are the energy barriers for the forward reaction and reverse reaction, respectively. Accordingly, the force-free forward and reverse reaction rates of the mechanophore under no applied force can be expressed as (Kramers, 1940)

$$
\begin{aligned}
& k^{f 0}=A \exp \left(-\frac{\Delta G^{S}}{k_{B} T}\right) \\
& k^{r 0}=B \exp \left(-\frac{\Delta G^{M}}{k_{B} T}\right)
\end{aligned}
$$

where the coefficients $A$ and $B$ are related to the rates at which the respective reactants are perturbed to the transition state (Bustamante et al., 2004; Hänggi et al., 1990; Kramers, 1940). Equation 21 further gives that the typical time scales for the force-free forward and reverse reactions of a mechanophore under no applied force are on the orders of $1 / k^{f 0}$ and $1 / k^{r 0}$, respectively. Since the forward and reverse reaction rates are on the order of $k^{f 0} \sim 10^{-7} s^{-1}$ and $k^{r 0} \sim 10^{-5} s^{-1}$ (Davis et al., 2009; Silberstein et al., 2014; Silberstein et al., 2013; Wang et al., 2014a), the time scale for mechanophores to reach chemical equilibrium under no applied force is $10^{5} \sim 10^{7} \mathrm{~s}$. Under applied forces, the time scale to reach chemical equilibrium can be significantly shortened.

As illustrated in Fig. 5b, an applied force $f$ on the mechanopore will vary the energy barriers of the forward reaction and reverse reaction to $\Delta G^{S}-f \Delta x^{S}$ and $\Delta G^{M}+f \Delta x^{M}$, respectively; where $\Delta x^{S}$ and $\Delta x^{M}$ are the effective distances between the reactant states (i.e., spriropyran and merocyanine) and the transition state along the reaction coordinate, respectively (Bustamante et al., 2004). When the MCR elastomer is deformed, the applied force on the mechanopores in the ith network is $f_{i}$, given by Eq. 7 . Therefore, the forward and reverse reaction rates of mechanophores on the ith network of a deformed 
MCR elastomer can be expressed as (Bell, 1978; Kauzmann and Eyring, 1940; Ribas-Arino and Marx, 2012)

$$
\begin{gathered}
k_{i}^{f}=A \exp \left[-\frac{\left(\Delta G^{S}-f_{i} \Delta x^{S}\right)}{k_{B} T}\right]=k^{f 0} \exp \left(\frac{f_{i} \Delta x^{S}}{k_{B} T}\right) \\
k_{i}^{r}=B \exp \left[-\frac{\left(\Delta G^{M}+f_{i} \Delta x^{M}\right)}{k_{B} T}\right]=k^{r 0} \exp \left(-\frac{f_{i} \Delta x^{M}}{k_{B} T}\right)
\end{gathered}
$$

Equation 22a gives that the typical forces to induce significant forward reactions of mechanophores are on the order of $\Delta G^{S} / \Delta x^{S} \sim 10^{-9} N$. The coefficients for reactions of mechanophores, (i.e., $k^{f 0}$, $k^{r 0}, \Delta x^{S}$ and $\Delta x^{M}$ ) can be measured by single-molecule force microscopy (Wang et al., 2014a) and calculated by atomic or molecular-dynamic calculations (Davis et al., 2009; Silberstein et al., 2014; Silberstein et al., 2013). While $k^{f 0}$ and $k^{r 0}$ can have very different values, $\Delta x^{S}$ and $\Delta x^{M}$ should be on the same order as reported previously (Silberstein et al., 2014; Silberstein et al., 2013). For simplicity of analysis in the current study, we further assume

$$
\Delta x^{M}=\Delta x^{S}=\Delta x
$$

where $\Delta x$ is taken as the effective geometry distance between the reactant states (i.e., spriropyran and merocyanine) and the transition state.

\subsubsection{Merocyanine concentration at the equilibrium state}

Now that the dependence of the forward and reverse reaction rates on applied forces of polymer chains has been established, we will discuss the merocyanine concentration in the MCR elastomer. If the 
chain forces are maintained constants over a period of time long enough, the chemical reaction of mechanophores can reach equilibrium. At the equilibrium state, Eq. 17 reduces to

$$
k_{i}^{f} c_{i}^{S}-k_{i}^{r} c_{i}^{M}=0
$$

Therefore, the amount of spiropyrans and merocyanines on the ith network per unit volume of the MCR elastomer at the current equilibrium state can be calculated as

$$
\begin{gathered}
c_{i}^{S}=\frac{k_{i}^{r}}{k_{i}^{r}+k_{i}^{f}} c_{i} \\
c_{i}^{M}=\frac{k_{i}^{f}}{k_{i}^{r}+k_{i}^{f}} c_{i}
\end{gathered}
$$

in which $k_{i}^{f}$ and $k_{i}^{r}$ are constants at equilibrium state, owning to the constant chain forces.

Furthermore, a combination of Eqs. 20, 22 and 25b gives the number of merocyanines in a unit volume of the MCR elastomer at equilibrium state as

$$
c^{M}=c \sum_{i=1}^{m}\left[\frac{V_{i} k^{f 0}}{k^{r 0} \exp \left(\frac{-2 f_{i} \Delta x}{k_{B} T}\right)+k^{f 0}}\right]
$$

where the force applied by polymer chains, $f_{i}$, is related to the deformation of the MCR elastomer by Eq. 7; and the volume ratio of the ith network in the MCR elastomer, $V_{i}$, is given by Eq. 4. 


\subsubsection{Transient evolution of merocyanine concentration}

The chain forces $f_{i}$ in the MCR elastomer can be varied upon deformation of the elastomer; and consequently the forward and reverse reaction rates, $k_{i}^{f}$ and $k_{i}^{r}$, will be changed over time according to Eq. 22. As a result, the merocyanine concentration $c_{i}^{M}$ will evolve over time, until a new equilibrium state is achieved. In this section, we will discuss the transient evolution of merocyanine concentration due to various variations of chain forces.

\section{Case 1: Constant chain force after a sudden change}

If the chain forces are maintained constant after a sudden change at time $t_{0}$, the chemical reaction rates $k_{i}^{f}$ and $k_{i}^{r}$ will be independent of time after $t_{0}$. According to Eq. 19, the evolution of merocyanine concentration in the $i t h$ network after $t_{0}$ can be calculated as

$$
c_{i}^{M}(t)=\exp \left[-\left(k_{i}^{f}+k_{i}^{r}\right) t\left[\left.c_{i}^{M}\right|_{t=t_{0}}-\frac{c_{i} k_{i}^{f}}{k_{i}^{f}+k_{i}^{r}}\right]+\frac{c_{i} k_{i}^{f}}{k_{i}^{f}+k_{i}^{r}}\right.
$$

where $k_{i}^{f}$ and $k_{i}^{r}$ are the time-independent reaction rates given by Eq. 22 with the chain forces applied at $t_{0}$.

We will discuss two representative examples for the case of constant chain forces after sudden changes. In the first example (Fig. 6a-c), the chain force is initially zero and suddenly changed to and maintained as a non-zero constant value, i.e.,

$$
f_{i}(t)=\left\{\begin{array}{l}
0, t \leq 0 \\
f, t>0
\end{array}\right.
$$

According to Eqs. 25 and 27, the merocyanine concentration in the ith network can be calculated as 


$$
\begin{aligned}
c_{i}^{M}(t)= & c_{i} \frac{k^{f 0}}{k^{r 0}+k^{f 0}}, t \leq 0 \\
c_{i}^{M}(t)= & c_{i}\left[\frac{k^{f 0}}{k^{f 0}+k^{r 0}}-\frac{k^{f 0} \exp \left(\frac{f \Delta x}{k_{B} T}\right)}{k^{f 0} \exp \left(\frac{f \Delta x}{k_{B} T}\right)+k^{r 0} \exp \left(\frac{-f \Delta x}{k_{B} T}\right)}\right] \exp \left\{-t\left[k^{f 0} \exp \left(\frac{f \Delta x}{k_{B} T}\right)+k^{r 0} \exp \left(\frac{-f \Delta x}{k_{B} T}\right)\right]\right\} \\
& +\frac{c_{i} k^{f 0} \exp \left(\frac{f \Delta x}{k_{B} T}\right)}{k^{f 0} \exp \left(\frac{f \Delta x}{k_{B} T}\right)+k^{r 0} \exp \left(\frac{-f \Delta x}{k_{B} T}\right)}, t>0
\end{aligned}
$$

where $k^{f 0}$ and $k^{r 0}$ are given by Eq. 21. We plot Eq. 29 with different levels of suddenly applied chain forces in Fig. 6b. It can be seen that the merocyanine concentration increases monotonically to new equilibriums over different time scales corresponding to the different applied chain forces. Based on Eq. 29, the time scale for merocyanine to reach the new equilibrium concentration can be approximated as

$$
t_{e q} \approx \frac{1}{k^{f 0} \exp \left(\frac{f \Delta x}{k_{B} T}\right)+k^{r 0} \exp \left(\frac{-f \Delta x}{k_{B} T}\right)}
$$

From Fig. 6c, it can be seen that the $t_{e q}$ given by Eq. 30 is a non-monotonic function of the suddenly applied chain force. The required time features a maximum value of $\sim 2.54 \times 10^{5}$ s at $f \Delta x / k_{B} T \approx 2.54$. As the applied chain force further increases, the required time decreases drastically. For example, as $f \Delta x / k_{B} T$ reaches 15 , the required time scale reduces to $\sim 1 \mathrm{~s}$.

In the second example (Fig. 6d-f), the chain-force is initially maintained to be a non-zero constant value and then suddenly relaxed to zero, i.e., 


$$
f_{i}(t)=\left\{\begin{array}{l}
f, t \leq 0 \\
0, t>0
\end{array}\right.
$$

According to Eqs. 25 and 27, the merocyanine concentration in the ith network can be further calculated as

$$
\begin{aligned}
& c_{i}^{M}(t)=c_{i} \frac{k^{f 0} \exp \left(\frac{f \Delta x}{k_{B} T}\right)}{k^{f 0} \exp \left(\frac{f \Delta x}{k_{B} T}\right)+k^{r 0} \exp \left(\frac{-f \Delta x}{k_{B} T}\right)}, t \leq 0 \\
& c_{i}^{M}(t)=c_{i}\left[\frac{k^{f 0} \exp \left(\frac{f \Delta x}{k_{B} T}\right)}{k^{f 0} \exp \left(\frac{f \Delta x}{k_{B} T}\right)+k^{r 0} \exp \left(\frac{-f \Delta x}{k_{B} T}\right)}-\frac{k^{f 0}}{k^{f 0}+k^{r 0}}\right] \exp \left[-t\left(k^{f 0}+k^{r 0}\right)\right]+\frac{c_{i} k^{f 0}}{k^{f 0}+k^{r 0}}, t>0
\end{aligned}
$$

where $k^{f 0}$ and $k^{r 0}$ are given by Eq. 21. We plot Eq. 32 with different levels of initially applied chain forces in Fig. 6e. It can be seen that the merocyanine concentration decreases monotonically to a new equilibrium, $c_{i} k^{f 0} /\left(k^{f 0}+k^{r 0}\right)$, over the same time scale, independent of the initial chain forces. Based on Eq. 32, the time scale for merocyanine to reach the new equilibrium concentration can be approximated as

$$
t_{e q} \approx \frac{1}{k^{f 0}+k^{r 0}}
$$

which is approximately $\sim 5 \times 10^{4} \mathrm{~s}$ (Fig. 6f).

\section{Case 2: Time-dependent chain forces}


If the chain force $f_{i}$ varies with time after the initial time $t_{0}$, the chemical reaction rates $k_{i}^{f}$ and $k_{i}^{r}$ will also depend on time after $t_{0}$. In this case, the merocyanine concentration can be calculated through a combination of Eqs. 19, 20 and 22, given that the time-dependent chain force in Eq. 22 is known. Here we study one example, where the chain force gradually increases with a constant rate over time (Fig. 6g), i.e.,

$$
f_{i}(t)=\left\{\begin{array}{l}
0, t \leq 0 \\
r \cdot t, t>0
\end{array}\right.
$$

where the loading rate of chain force $r$ has a dimension of $N \cdot s^{-1}$. Based on Eqs. 19 and 22 , the variation of merocyanine concentration in the ith network over time can be calculated as

$$
\begin{gathered}
c_{i}^{M}(t)=c_{i} \frac{k^{f 0}}{k^{r 0}+k^{f 0}}, t \leq 0 \\
c_{i}^{M}(t)=c_{i} \exp \left[\frac{\left.-k^{f 0} \exp \left(\frac{r t \Delta x}{k_{B} T}\right)+k^{r 0} \exp \left(\frac{-r t \Delta x}{k_{B} T}\right)\right]}{\left.\frac{r \Delta x}{k_{B} T}\right]}\right] \\
\left\{\int_{0}^{t} k^{f 0} \exp \left[\frac{k^{f 0} \exp \left(\frac{r \tau \Delta x}{k_{B} T}\right)-k^{r 0} \exp \left(\frac{-r \tau \Delta x}{k_{B} T}\right)}{\frac{r \Delta x}{k_{B} T}}+\frac{r \tau \Delta x}{k_{B} T}\right] d \tau+\frac{k^{f 0}}{k^{f 0}+k^{r 0}} \exp \left(\frac{k^{f 0}-k^{r 0}}{\frac{r \Delta x}{k_{B} T}}\right)\right\}, t>0
\end{gathered}
$$

which is plotted in Fig. 6h for different loading rates of the chain force. It can be seen that the merocyanine concentration on the ith network initially maintains at $c_{i} k^{f 0} /\left(k^{f 0}+k^{r 0}\right)$ and then gradually increases to approximately the fully-activated concentration $c_{i}$ with the rising chain force. Based on Eq. 35, the time scale for merocyanine to approximate the fully-activated concentration can be 
calculated and plotted in Fig. 6I. It is evident that a higher loading rate requires a shorter time $t_{\text {eq }}$ to fully activate the mechanophore.

\subsubsection{Fluorescent intensity of the MCR elastomer}

Now that the concentration of merocyanine in the MCR elastomer can be determined at both equilibrium and transient states, we will correlate the merocyanine concentration with the fluorescent intensity of the elastomer observed in experiments. We assume that the observed fluorescent intensity of an MCR-elastomer film is proportional to the amount of merocyanine molecules throughout the thickness of the film (Wang et al., 2014b). Therefore, the fluorescence intensity of the elastomer film at the deformed state can be expressed as

$$
Q=\operatorname{const} \cdot c^{M} h
$$

where $Q$ and $h$ are the fluorescence intensity and thickness of the MCR elastomer film at the current states, respectively; and const is a constant independent of the deformation of the elastomer. The fluorescence intensity of the elastomer film at undeformed state can be expressed as $Q_{0}=c o n s t \cdot c^{M 0} h_{0}$,

where $c^{M 0}=c^{M}\left(\Lambda_{i}=1\right)$ and $h_{0}$ is the film thickness at the undeformed state. In this way, we can quantitatively correlate the macroscopic stress-strain behavior and florescent intensity of MCR elastomer with the microscopic chain forces in polymer networks and activation of mechanophores.

\section{Results of the theoretical model compared with experiments}

Section 3 presents an interpenetrating-network model for MCR elastomers with non-uniform chain lengths. The model can adopt different chain-length distribution functions, $p_{i}\left(n_{i}\right)$ in Eq. 2. In this 
section, we will use the model with different chain-length distributions to discuss the macroscopic stressstrain behaviors of the MCR elastomers and microscopic chemical reactions of the mechanophores in the elastomers.

\subsection{Chain-length distributions}

\subsubsection{Dirac delta distribution}

If all chains in the MCR elastomer have the same chain length, the chain probability density follows the Dirac delta distribution (Fig. 7a), expressed as

$$
p_{i}\left(n_{i}\right)= \begin{cases}1 & n_{i}=n_{*} \\ 0 & \text { else }\end{cases}
$$

where $n_{*}$ denotes the chain-length (Kuhn link number) of each chain.

\subsubsection{Uniform distribution}

If the chains with different chain-lengths have equal chain concentration, the chain probability density can be expressed by the uniform distribution (Fig. 7c), i.e.,

$$
p_{i}\left(n_{i}\right)= \begin{cases}\frac{1}{b-a} & a \leq n_{i} \leq b \\ 0 & \text { else }\end{cases}
$$

\subsubsection{Normal distribution (Gaussian distribution)}

If the chain-length is distributed normally around an average value, the chain probability density can be expressed by the normal distribution (Fig. 7e), i.e.,

$$
p_{i}\left(n_{i}\right)=\frac{1}{\sqrt{2 \pi} \delta} \exp \left[-\frac{\left(n_{i}-n_{0}\right)^{2}}{2 \delta^{2}}\right]
$$

Where $n_{0}$ and $\delta$ are the mean and standard deviation of $n_{i}$, respectively. 


\subsubsection{Log-normal distribution}

If the logarithm of the chain-length is normally distributed, the chain probability density follows a log-normal distribution (Fig. 7g), written as,

$$
p_{i}\left(n_{i}\right)=\frac{1}{n_{i} \sqrt{2 \pi} \delta} \exp \left[-\frac{\left(\ln n_{i}-\ln n_{0}\right)^{2}}{2 \delta^{2}}\right]
$$

where $\ln n_{0}$ and $\delta$ are the mean and standard deviation of $\ln n_{i}$.

\subsubsection{Weibull distribution}

The chain probability density can also be expressed as the Weibull distribution (Fig. 7i), i.e.,

$$
p_{i}\left(n_{i}\right)=\frac{a}{b}\left(\frac{n_{i}}{b}\right)^{a-1} e^{-\left(\frac{n_{i}}{b}\right)^{a}}
$$

where $a>0$ is the shape parameter and $b>0$ is the scale parameter.

It should be noted that, by normalization, we ensure the summation of the probability densities is equal to a unit in all types of chain-length distributions, i.e., $\sum_{i=1}^{m} p_{i}\left(n_{i}\right)=1$.

\subsection{Macroscopic stress-strain behavior}

By adopting a number of chain-length distributions given in Section 4.1, we compare the theoretical prediction and experimental results of stress-strain behaviors of the MCR elastomers under pure-shear uniaxial tension. Here, we show that the experimental stress-strain behaviors can be well predicted by our model with various chain-length distributions. 
If the chain-length distribution follows the Dirac delta distribution (Eq. 37), the polymer network only contains one type of chains with the same chain-length. The uniaxial nominal stress-stretch relation under the pure-shear condition (Eq. 15) can be reduced to

$$
s_{1}=\left(\lambda-\frac{1}{\lambda^{3}}\right) \frac{N \sqrt{n_{*}} k_{B} T \beta_{*}}{3 \Lambda_{*}}
$$

where $N$ is the total polymer chain number in a unit volume of the elastomer, $n_{*}$ is the chain link number of this only chain type, $\beta_{*}=L^{-1}\left(\Lambda_{*} / \sqrt{n_{*}}\right), \Lambda_{*}$ is the chain stretch that can be calculated as $\Lambda_{*}=\lambda^{2}+\lambda^{-2}+1$, and $\lambda$ is the macroscopic stretch along the stretching direction. As shown in Fig. 7ab, the predicted stress-stretch curve matches well with the experiments. It should be noted that, when the chain-length follows the Dirac delta distribution, our network model is equivalent to the Arruda-Boyce model (Arruda and Boyce, 1993).

If the chain-length distribution follows a uniform distribution (Eq. 38), the network contains a number of types of polymer chains, each type with equal probability density. From Fig. 7d, we can see that the uniform distribution can well predict the initial potion of the stress-stretch curve, but fails to describe the stiffening part, which indicates that the chain lengths within the elastomer should be nonuniformly distributed.

If we assume polymer chains with inhomogeneous chain-lengths, we can describe the chainlength distribution with normal distribution (Eq. 39), log-normal distribution (Eq. 40), Weibull distribution (Eq. 41) and others. As illustrated in Figs. 7b, 7f, 7h, and 7j, the model with fitted parameters for different types of chain-length distributions can all match the experimentally measured stress-stretch data of MCR elastomer consistently. This means MCR elastomers with different chainlength distributions can exhibit similar stress-strain behaviors. 


\subsection{Microscopic chemical reactions of mechanophores and macroscopic fluorescence intensity}

Next, we calculate the fluorescent intensity of the deformed MCR elastomer films and compare the theoretical predictions with experimental results discussed in Sec. 2. Since the fluorescent intensities of the deformed MCR elastomer films are measured at the equilibrium states, we will focus the calculation on the equilibrium states as discussed in Sec. 3.2.3. Given a macroscopic deformation state $\left(\lambda_{1}, \lambda_{2}\right.$, and $\left.\lambda_{3}\right)$, the forces applied on mechanophores in the $i$ th network can be calculated by Eq. 7 , the equilibrium concentration of merocyanines in the MCR elastomer by Eq. 25b, and the fluorescent intensity of the MCR elastomer by Eq. 36. Here we take the link length (or Kuhn length) of the polymer chains of PDMS to be $l=5.2 \times 10^{-10} \mathrm{~m}$ (Evmenenko et al., 2005), and the forward and reverse reaction rates to be $k^{f 0}=1.94 \times 10^{-7} s^{-1}$ and $k^{r 0}=1.99 \times 10^{-5} s^{-1}$, respectively, which were obtained from molecular dynamic calculations (Davis et al., 2009; Silberstein et al., 2014; Silberstein et al., 2013). The effective geometry distance $\Delta x$ is taken as a fitting parameter in the current model, but the value of $\Delta x$ is on the order of $10^{-10} \mathrm{~m}$, as previously reported (Davis et al., 2009; Silberstein et al., 2014; Silberstein et al., 2013; Wang et al., 2014a). Based on the model, we find that the microscopic chemical reactions of mechanophores and macroscopic fluorescence intensity of the MCR elastomers at equilibrium states are significantly affected by the deformation of the MCR elastomers, effective reaction distance $\Delta x$, and polymer chain-length distribution.

The equilibrium concentration of merocyanines increases monotonically with the applied stretch $\lambda$ (Figs. 8a, 8c and 8e), because the forces applied by polymer chains on mechanophores, which promote the transition from spiropyran to merocyanines, increases monotonically with the applied stretch. By considering the thickness effect involved in the observed region (Eq. 36, discussed in Sec. 2), the observed fluorescence intensity first decreases slightly and then increases proportionally with the applied stretch (Figs. 8b, 8d, 8f and 3c). 
The emitted fluorescence intensity can be affected by the effective reaction distance $\Delta x$ which is aligned with the applied force during the chemical reaction. As effective distance $\Delta x$ increases, the same chain force leads to greater mechanically-induced reduction of the activation energy of the chemical reaction. As a result, the forward chemical reaction (from spiropyran to merocyanine) becomes stronger, inducing higher fluorescence intensity (Fig. 8b).

The fluorescence intensity ratio of the MCR elastomer is also significantly affected by the function of chain-length distribution. Under the same deformation, the chain force decreases monotonically with the link number in a network (Fig. 9a). Given a non-uniform chain-length distribution, the chain forces applied to mechanophores and thus the extent of activation of mechanophores will be non-uniformly distributed based on the function of chain-length distribution (Fig. 9b). For example, if we consider the chain-length distribution as a log-normal function, whose shape is determined by the mean $\left(\ln n_{0}\right)$ and standard deviation $(\delta)$ of the logarithm of the chain length $\left(\ln n_{i}\right)$. As the mean chain length increases, the resultant longer chains will exert lower forces on mechanophores under the same stretch (Fig. 9a), so that less merocyanines will be present. Therefore, the emitted fluorescence intensity decreases with the increase of mean chain length (Fig. 8d). As the standard deviation $\delta$ increases, the number of short chains in the elastomer increases (Fig. 7g); therefore, the emitted fluorescence intensity increases with the rise of standard deviation $\delta$ (Fig. 8f).

In particular, it should be noted that even though microphysical models with different chainlength distributions can give similar macroscopic stress-strain curves (Figs, 7b, 7f, 7h, 7j and 10a), the predicted fluorescence intensities can be significantly different from one other (Fig. 10bc). As discussed in Sec. 4.2, the microphysical models which consider chain-length distribution as Derac Delta, normal, log-normal and Weibull distributions can predict similar macroscopic stress-strain curves, all consistent with the experimentally measured stress-strain curve (Figs, 7b, 7f, 7h, 7j and 10a). However, the predicted fluorescence intensity (Fig. 10bc) resulting from different chain length distributions differs 
drastically with one another. We further find that the predicted fluorescence by the model with log-normal distribution can match experimental observations consistently (Fig. 10bc) by setting the total chain number density as $N=8 \times 10^{25}$, the mean and standard deviation of the logarithm of the chain-length as $\ln n_{0}=\ln 14$ and $\delta=0.8$, and the effective reaction distance as $\Delta x=4 \times 10^{-10} \mathrm{~m}$. The effective distance $\Delta x$ falls within in a reasonable range compared with that obtained from the molecular dynamic computations in (Davis et al., 2009; Silberstein et al., 2014). As shown in Fig. 10bc, the predicted fluorescence intensity by the model with log-normal distribution can match the experimental data for both uniaxial pure-shear tension and equibiaxial tension, and varied spiropyran concentrations $(0.05 \%$ to $0.5 \%$ $w t)$.

\section{Potential applications of MCR elastomers compared with finite-element model}

In this section, we will first demonstrate a few potential applications of MCR elastomers, where we generate fluorescence patterns by deforming the MCR elastomers in designed structures. To model the complicated deformations associated with the fluorescence patterns, we program the microphysical model developed in Sec. 3 into an ABAQUS UHYPER user subroutine (see Appendix B for more details). Based on the calculated deformation patterns, the fluorescence intensity can be predicted by Eq. $\mathbf{3 6}$ and compared with experimental results.

\subsection{Strain imaging}

Strain imaging has been intensively studied by using photoelastic materials that are usually rigid materials (Frocht, 1941). For highly deformable elastomers, the strain maps have typically been characterized by digital image correlation methods (Hall et al., 2012; Maskarinec et al., 2009; Peters and Ranson, 1982; Xu et al., 2010). More recently, Ducrot et al. used a luminescent emission of the MCR dioxetane molecules to detect the fracture of sacrificial bonds in double-network polymers (Ducrot et al., 2014). Here, we demonstrate that MCR elastomers can provide a nondestructive method for strain imaging by observing the fluorescence intensities of highly deformed MCR elastomers. 
The first example is fluorescence emission around a circular hole of a uniaxially deformed MCR elastomer. We first cut a circular hole with a diameter of $2 \mathrm{~mm}$ in the MCR film (thickness $\sim 300 \mu \mathrm{m})($ Fig. 11a), and then uniaxially stretch the MCR film by $\lambda=1.7$ under a plane-stress condition. As a result, the circular hole deforms into an ellipse. The observed fluorescence intensities around the minor axes are much stronger than other regions on the MCR film (Fig. 11b). We can further convert the fluorescence image into the first-invariant mapping (Fig. 11c) by using the relationship between the elastomer deformation and the fluorescence intensity predicted by Eq. 36. At the same time, using the finite-element model with UHYPER user subroutine, we can compute the first invariant around the deformed elliptic holes as shown in Fig. 11d. The two-dimensional model is discretized by CPE4R elements, and the accuracy of the model is ascertained through mesh refinement studies. From Figs. 11c-e, the experimentally observed and the predicted first-invariant mappings match with each other qualitatively and quantitatively. As another example, we cut a notch in the MCR film and stretch the film uniaxially by $\lambda=1.4$ (Fig. 12ab). As illustrated in Figs. 12c-e, the observed and predicted fluorescence intensities and first-invariant fields also match each other fairly well. Through these two examples, we demonstrate that MCR elastomers have the potential to be used as photo-hyperealstic materials that can quantitatively map the deformation (e.g., first invariant) of highly deformed elastomers. While conventional digital image correlation methods may provide accurate full-field measurements (Hall et al., 2012; Maskarinec et al., 2009; Peters and Ranson, 1982; Xu et al., 2010), they can only characterize the surface strains of measured materials. In contrast, the current method can provide information about the internal deformation or damage (Ducrot et al., 2014) of materials undergoing large deformation and fracture.

\subsection{Color/fluorescence display}

The MCR elastomers can also be deformed in designed patterns to display colors or fluorescence patterns. Various methods have been exploited to display color or fluorescence on elastomer systems, such as coupling rigid light-emitting diodes with elastomeric matrix (Jeong et al., 2012; Kim et al., 2011; 
Kim et al., 2010; Park et al., 2009; Sekitani et al., 2009), using electro-luminescent elastomers (Filiatrault et al., 2012; Liang et al., 2013; White et al., 2013; Yu et al., 2011) and filling elastomer channels with pigment fluids (Hagedon et al., 2012; Heikenfeld et al., 2009; Morin et al., 2012; Shepherd et al., 2011; Yeo et al., 2010). Wang et al. has demonstrated that MCR elastomers can be assembled into an electrocontrollable panel to display on-demand fluorescence and colors (Wang et al., 2014b). To test the applicability of the model, we investigate here how an MCR elastomer can be used to display fluorescence patterns by simple mechanical contacts (Gossweiler et al., 2014).

We fabricate an embossed mask with letters "DUKE" by printing a thermoplastic PLA (Formfutura, Netherland) with a 3D printer MakerBot Replicator 2 (MakerBot, US) (Lin et al., 2014). The size of each letter is around $2 \mathrm{~mm}$ by $2 \mathrm{~mm}$ in area and $1 \mathrm{~mm}$ in depth. We then push the embossed mask on into an MCR elastomer $(\sim 800 \mu \mathrm{m})$ to a depth of around $400 \mu \mathrm{m}$ (Fig. 13a). The shear modulus of the thermoplastic PLA is $\sim 2 \mathrm{MPa}$, while the shear modulus of the MCR elastomer is much lower, $\sim 140 \mathrm{kPa}$ (Wang et al., 2014b). Around the contact regions, large deformations are generated in the MCR elastomer which reveal increased fluorescence intensity from activated mechanophores. The fluorescence patterns, observed from the bottom of the MCR elastomer, can clearly replicate the letter patterns of the mask (Fig.

13b). We further simulate this contact problem in the finite-element program ABAQUS with the UHYPER subroutine. The rigid mask is modeled as a linear elastic material with Young's modulus of 6 Mpa. As illustrated in Fig. 13cd, the simulated letter patterns can quantitatively match the observed the fluorescence patterns.

\section{Conclusive remarks}

In summary, we present a constitutive model for mechanochemically responsive elastomers to quantitatively reveal how the macroscopic deformation of the elastomer translates to the molecular forces in polymer networks with non-uniform chain lengths, and how the chain forces subsequently affect 
chemical reactions of mechanophores coupled to the networks. The predicted macroscopic stress-stretch behaviors and the fluorescence emission of deformed MCR elastomers match consistently with experimental observations. In particular, we quantitatively explain that

1). MCR elastomers with time-independent stress-strain behaviors can present time-dependent variation of color or fluorescence due to the kinetics of mechanophore activation; and

2). MCR elastomers with different chain-length distributions exhibit similar stress-strain behaviors, but very different colors/fluorescence under the same deformation - indicating the importance of chain-length distribution in affecting chemical reactions of mechanophores.

The theoretical model suggests strategies for designing more complex polymer network architectures to achieve better mechanochemical activations of various mechanophores (Beyer and Clausen-Schaumann, 2005; Black et al., 2011a; Caruso et al., 2009; Kean and Craig, 2012). As discussed in Sec. 4, the activations of forward reactions of mechanophores attached to shorter network chains are easier than on the longer chains under the same stretch. However, an elastomer with only short-chain network may suffer from low stretchability and low toughness. Therefore, it may be desirable to design bimodal MCR elastomers or gels with long-chain and short-chain networks interpenetrated with each other (Ducrot et al., 2014; Gong et al., 2003; Sun et al., 2012), where the mechanophores are only coupled to the short-chain network. Deformation of the resultant elastomers or gels will highly stretch (and even fracture) the short-chain network to effectively activate the forward reactions; meanwhile, the long-chain network will maintain the integrity of the elastomers or gels under deformation (Ducrot et al., 2014; Gong et al., 2003; Sun et al., 2012; Zhao, 2012). As shown in Fig. 14, this type of double-network MCR elastomer is expected to achieve much higher activation of the mechanophores than the usual MCR elastomers with non-uniform chain-lengths. Mechanophores may also be coupled to other nonconventional network architectures for the design of high-efficient MCR elastomers and gels (Zhao, 2014). 
We further program the constitutive model into finite-element models, through which we demonstrate the MCR elastomers' potential applications for strain imaging and color/fluorescence displays. These models can further facilitate the design of MCR polymers in the macro/micro structure level to create MCR devices for diverse applications, such as sensors, memories, flexible displays, optoelectronics, biomedical luminescent devices and dynamics camouflage skins (Beyer and ClausenSchaumann, 2005; Black et al., 2011a; Caruso et al., 2009; Chi et al., 2012; Kean and Craig, 2012; Sagara and Kato, 2009; Wang et al., 2014b).

\section{Appendix A. Worm-Like-Chain model}

If we assume a polymer chain in the ith network follows the Worm-Like-Chain model, the strain energy of this chain can be expressed as (Bischoff et al., 2002a; Bischoff et al., 2002b; Kuhl et al., 2005; Ogden et al., 2006)

$$
w_{i}=\frac{k_{B} T n_{i} l}{4 P_{e}}\left[2\left(\frac{r_{i}}{n_{i} l}\right)^{2}+\frac{1}{1-r_{i} /\left(n_{i} l\right)}-\frac{r_{i}}{n_{i} l}\right]+w_{0}
$$

where $r_{i}$ is the current end to end distance, $n_{i} l$ is the contour length, $P_{e}$ is the persistence length and $w_{0}$ is a constant energy at the undeformed state. The chain force can be derived as (Bouchiat et al., 1999; Marko and Siggia, 1995)

$$
f_{i}=\frac{\partial w_{i}}{\partial r_{i}}=\frac{k_{B} T}{4 P_{e}}\left[\frac{4 r_{i}}{n_{i} l}+\frac{1}{\left(1-r_{i} /\left(n_{i} l\right)\right)^{2}}-1\right]
$$




\section{Appendix B. Formulations for user subroutine UHYPER}

To model a nearly incompressible MCR elastomer in the ABAQUS, we modify the free energy of the elastomer network in Eq. 10 by adding a tail term, i.e.,

$$
W=\sum_{i=1}^{m} W_{i}+\frac{(J-1)^{2}}{D}=\sum_{i=1}^{m}\left[N_{i} n_{i} k_{B} T\left(\frac{\beta_{i}}{\tanh \beta_{i}}+\ln \frac{\beta_{i}}{\sinh \beta_{i}}\right)\right]+\frac{(J-1)^{2}}{D}
$$

where the volume change is $J=\operatorname{det} \mathbf{F}, \mathbf{F}$ is the deformation gradient tensor, and $D$ is a constant parameter that should be a near zero value to enforce the nearly incompressible condition.

In the user subroutine UHYPER, derivatives of the free energy $W$ over strain invariants and volume change $J$ should be also explicitly defined. The non-zero terms include the following.

$$
\frac{\partial W}{\partial \bar{I}_{1}}=\sum_{i=1}^{m}\left[\frac{N_{i} \sqrt{n_{i}} k_{B} T}{\sqrt{12}} \beta_{i} \bar{I}_{1}^{-1 / 2} J^{1 / 3}\right]
$$

where $\bar{I}_{1}=J^{-2 / 3} I_{1}$.

$$
\begin{gathered}
\frac{\partial W}{\partial J}=\sum_{i=1}^{m}\left[\frac{N_{i} \sqrt{n_{i}} k_{B} T}{\sqrt{27}} \beta_{i} \bar{I}_{1}^{-1 / 2} J^{-2 / 3}\right]+\frac{2(J-1)}{D} \\
\frac{\partial^{2} W}{\partial \bar{I}_{1}^{2}}=\sum_{i=1}^{m} \frac{N_{i} \sqrt{n_{i}} k_{B} T}{\sqrt{12}} J^{1 / 3}\left(\frac{1}{\sqrt{12 n_{i}}} \frac{\bar{I}_{1}^{-1} J^{1 / 3}}{\left(\frac{-1}{\sinh ^{2} \beta_{i}}+\frac{1}{\beta_{i}^{2}}\right)}-\frac{1}{2} \beta_{i} \bar{I}_{1}^{-3 / 2}\right)
\end{gathered}
$$




$$
\begin{gathered}
\frac{\partial^{2} W}{\partial J^{2}}=\sum_{i=1}^{m} \frac{N_{i} \sqrt{n_{i}} k_{B} T}{\sqrt{27}} \bar{I}_{1}^{-1 / 2}\left[-\frac{2}{3} \beta_{i} J^{-5 / 3}+\frac{1}{\sqrt{27 n_{i}}} \frac{\bar{I}_{1}^{1 / 2} J^{-4 / 3}}{\left.\frac{-1}{\sinh ^{2} \beta_{i}}+\frac{1}{\beta_{i}^{2}}\right)}\right]+\frac{2}{D} \\
\frac{\partial^{2} W}{\partial \bar{I}_{1} \partial J}=\sum_{i=1}^{m} \frac{N_{i} \sqrt{n_{i}} k_{B} T}{\sqrt{12}}\left[\frac{1}{3} \beta_{i} J^{-2 / 3} \bar{I}_{1}^{-1 / 2}+\frac{1}{\sqrt{27 n_{i}}} \frac{J^{-1 / 3}}{\left(\frac{-1}{\sinh ^{2} \beta_{i}}+\frac{1}{\beta_{i}^{2}}\right)}\right]
\end{gathered}
$$

$$
\frac{\partial^{3} W}{\partial \bar{I}_{1}^{2} \partial J}=\sum_{i=1}^{m} \frac{N_{i} \sqrt{n_{i}} k_{B} T}{\sqrt{12}}\left(\begin{array}{l}
\frac{\bar{I}_{1}^{-1}}{\sqrt{12 n_{i}}}\left(\frac{2 / 3 J^{-1 / 3}}{\left(\frac{-1}{\sinh ^{2} \beta_{i}}+\frac{1}{\beta_{i}^{2}}\right)}-\frac{\left(\frac{2 \cosh \beta_{i}}{\sinh ^{3} \beta_{i}}-\frac{2}{\beta_{i}^{3}}\right) \sqrt{\frac{\bar{I}_{1}}{27 n_{i}}}}{\left(\frac{-1}{\sinh ^{2} \beta_{i}}+\frac{1}{\beta_{i}^{2}}\right)^{3}}\right) \\
-\frac{1}{2} \bar{I}_{1}^{-3 / 2}\left(\frac{J^{-1 / 3}}{\left(\frac{-1}{\sinh ^{2} \beta_{i}}+\frac{1}{\beta_{i}^{2}}\right)} \sqrt{\frac{\bar{I}_{1}}{27 n_{i}}}+\frac{1}{3} \beta_{i} J^{-2 / 3}\right)
\end{array}\right)
$$

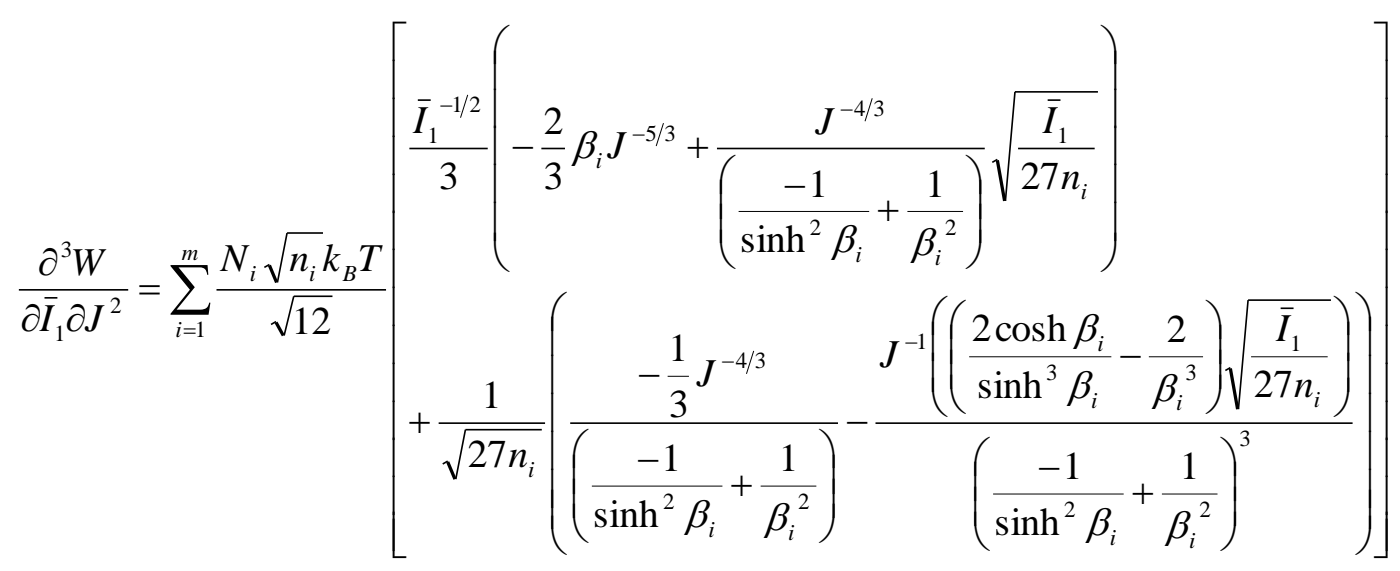




$$
\frac{\partial^{3} W}{\partial J^{3}}=\sum_{i=1}^{m} \frac{N_{i} \sqrt{n_{i}} k_{B} T}{\sqrt{27}}\left[\begin{array}{l}
\frac{-2}{3} I_{1}^{-1 / 2}\left(\frac{-5}{3} \beta_{i} J^{-8 / 3}+\sqrt{\frac{\bar{I}_{1}}{27 n_{i}}} \frac{J^{-7 / 3}}{\left(\frac{-1}{\sinh ^{2} \beta_{i}}+\frac{1}{\beta_{i}^{2}}\right)}\right) \\
\left.+\frac{1}{\sqrt{27 n_{i}}}\left(\frac{-\frac{4}{3} J^{-7 / 3}}{\left(\frac{-1}{\sinh ^{2} \beta_{i}}+\frac{1}{\beta_{i}^{2}}\right)}-\frac{\left.J^{-2}\left(\frac{2 \cosh \beta_{i}}{\sinh ^{3} \beta_{i}}-\frac{2}{\beta_{i}^{3}}\right) \sqrt{\frac{\bar{I}_{1}}{27 n_{i}}}\right)}{\left(\frac{-1}{\sinh ^{2} \beta_{i}}+\frac{1}{\beta_{i}^{2}}\right)^{3}}\right)\right]
\end{array}\right]
$$

The user subroutine UHYPER can be found in the supplementary data.

\section{Acknowledgement}

The work was supported by ONR (No. N00014-14-1-0528), NSF (No. CMMI-1253495, CMMI-1200515) and US Army Research Laboratory and the Army Research Office (No. W911NF-11-1-0071). 


\section{Reference}

Arruda, E.M., Boyce, M.C., 1993. A three-dimensional constitutive model for the large stretch behavior of rubber elastic materials. Journal of the Mechanics and Physics of Solids 41, 389-412.

Beiermann, B.A., Kramer, S.L.B., May, P.A., Moore, J.S., White, S.R., Sottos, N.R., 2014. The Effect of Polymer Chain Alignment and Relaxation on Force-Induced Chemical Reactions in an Elastomer.

Advanced Functional Materials 24, 1529-1537.

Bell, G.I., 1978. Models for the specific adhesion of cells to cells. Science 200, 618-627.

Beyer, M.K., Clausen-Schaumann, H., 2005. Mechanochemistry: the mechanical activation of covalent bonds. Chemical reviews 105, 2921-2948.

Bischoff, J., Arruda, E., Grosh, K., 2002a. A microstructurally based orthotropic hyperelastic constitutive law. Journal of applied mechanics 69, 570-579.

Bischoff, J., Arruda, E., Grosh, K., 2002b. Orthotropic hyperelasticity in terms of an arbitrary molecular chain model. Journal of applied mechanics 69, 198-201.

Black, A.L., Lenhardt, J.M., Craig, S.L., 2011a. From molecular mechanochemistry to stress-responsive materials. Journal of Materials Chemistry 21, 1655-1663.

Black, A.L., Orlicki, J.A., Craig, S.L., 2011b. Mechanochemically triggered bond formation in solid-state polymers. Journal of Materials Chemistry 21, 8460-8465.

Bouchiat, C., Wang, M., Allemand, J.-F., Strick, T., Block, S., Croquette, V., 1999. Estimating the persistence length of a worm-like chain molecule from force-extension measurements. Biophysical journal 76, 409-413.

Bustamante, C., Chemla, Y.R., Forde, N.R., Izhaky, D., 2004. Mechanical processes in biochemistry. Annual review of biochemistry 73, 705-748.

Caruso, M.M., Davis, D.A., Shen, Q., Odom, S.A., Sottos, N.R., White, S.R., Moore, J.S., 2009.

Mechanically-induced chemical changes in polymeric materials. Chemical reviews 109, 5755-5798.

Chen, Y., Spiering, A., Karthikeyan, S., Peters, G.W., Meijer, E., Sijbesma, R.P., 2012. Mechanically

induced chemiluminescence from polymers incorporating a 1,2-dioxetane unit in the main chain. Nature Chemistry 4, 559-562.

Chen, Y., Zhang, H., Fang, X., Lin, Y., Xu, Y., Weng, W., 2014. Mechanical Activation of Mechanophore Enhanced by Strong Hydrogen Bonding Interactions. ACS Macro Letters 3, 141-145.

Chi, Z., Zhang, X., Xu, B., Zhou, X., Ma, C., Zhang, Y., Liu, S., Xu, J., 2012. Recent advances in organic mechanofluorochromic materials. Chemical Society Reviews 41, 3878-3896.

Davis, D.A., Hamilton, A., Yang, J., Cremar, L.D., Van Gough, D., Potisek, S.L., Ong, M.T., Braun, P.V., Martínez, T.J., White, S.R., 2009. Force-induced activation of covalent bonds in mechanoresponsive polymeric materials. Nature 459, 68-72.

Ducrot, E., Chen, Y., Bulters, M., Sijbesma, R.P., Creton, C., 2014. Toughening elastomers with sacrificial bonds and watching them break. Science 344, 186-189.

Erman, B., Mark, J.E., 1997. Structures and properties of rubberlike networks. Oxford University Press New York.

Evmenenko, G., Mo, H., Kewalramani, S., Dutta, P., 2005. Conformational rearrangements in thin films of polydimethylsiloxane melt. arXiv preprint cond-mat/0510713.

Filiatrault, H.L., Porteous, G.C., Carmichael, R.S., Davidson, G.J., Carmichael, T.B., 2012. Stretchable

Light - Emitting Electrochemical Cells Using an Elastomeric Emissive Material. Advanced Materials 24, 2673-2678.

Flory, P.J., 1944. Network Structure and the Elastic Properties of Vulcanized Rubber. Chemical reviews 35, 51-75.

Flory, P.J., 1953. Principles of polymer chemistry. Cornell University Press.

Frocht, M.M., 1941. Photoelasticity. J. Wiley.

Gong, J.P., Katsuyama, Y., Kurokawa, T., Osada, Y., 2003. Double - Network Hydrogels with Extremely High Mechanical Strength. Advanced Materials 15, 1155-1158. 
Gossweiler, G.R., Hewage, G.B., Soriano, G., Wang, Q., Welshofer, G.W., Zhao, X., Craig, S.L., 2014. Mechanochemical Activation of Covalent Bonds in Polymers with Full and Repeatable Macroscopic Shape Recovery. ACS Macro Letters 3, 216-219.

Hagedon, M., Yang, S., Russell, A., Heikenfeld, J., 2012. Bright e-Paper by transport of ink through a white electrofluidic imaging film. Nature Communications 3, 1173.

Hall, M.S., Long, R., Hui, C.-Y., Wu, M., 2012. Mapping three-dimensional stress and strain fields within a soft hydrogel using a fluorescence microscope. Biophysical journal 102, 2241-2250.

Hänggi, P., Talkner, P., Borkovec, M., 1990. Reaction-rate theory: fifty years after Kramers. Reviews of Modern Physics 62, 251.

Heikenfeld, J., Zhou, K., Kreit, E., Raj, B., Yang, S., Sun, B., Milarcik, A., Clapp, L., Schwartz, R., 2009. Electrofluidic displays using Young-Laplace transposition of brilliant pigment dispersions. Nature Photonics 3, 292-296.

Hickenboth, C.R., Moore, J.S., White, S.R., Sottos, N.R., Baudry, J., Wilson, S.R., 2007. Biasing reaction pathways with mechanical force. Nature 446, 423-427.

James, H.M., Guth, E., 1943. Theory of the elastic properties of rubber. The Journal of Chemical Physics $11,455-481$.

Jeong, G.S., Baek, D.-H., Jung, H.C., Song, J.H., Moon, J.H., Hong, S.W., Kim, I.Y., Lee, S.-H., 2012.

Solderable and electroplatable flexible electronic circuit on a porous stretchable elastomer. Nature

Communications 3, 977.

Kauzmann, W., Eyring, H., 1940. The viscous flow of large molecules. Journal of the American Chemical Society 62, 3113-3125.

Kean, Z.S., Craig, S.L., 2012. Mechanochemical remodeling of synthetic polymers. Polymer 53, 10351048.

Kim, R.-H., Bae, M.-H., Kim, D.G., Cheng, H., Kim, B.H., Kim, D.-H., Li, M., Wu, J., Du, F., Kim, H.S., 2011. Stretchable, transparent graphene interconnects for arrays of microscale inorganic light emitting diodes on rubber substrates. Nano letters 11, 3881-3886.

Kim, R.-H., Kim, D.-H., Xiao, J., Kim, B.H., Park, S.-I., Panilaitis, B., Ghaffari, R., Yao, J., Li, M., Liu, Z., 2010. Waterproof AlInGaP optoelectronics on stretchable substrates with applications in biomedicine and robotics. Nature materials 9, 929-937.

Kingsbury, C.M., May, P.A., Davis, D.A., White, S.R., Moore, J.S., Sottos, N.R., 2011. Shear activation of mechanophore-crosslinked polymers. Journal of Materials Chemistry 21, 8381-8388.

Kramers, H.A., 1940. Brownian motion in a field of force and the diffusion model of chemical reactions. Physica 7, 284-304.

Kuhl, E., Garikipati, K., Arruda, E.M., Grosh, K., 2005. Remodeling of biological tissue: mechanically induced reorientation of a transversely isotropic chain network. Journal of the Mechanics and Physics of Solids 53, 1552-1573.

Kuhn, W., Grün, F., 1942. Beziehungen zwischen elastischen Konstanten und Dehnungsdoppelbrechung hochelastischer Stoffe. Kolloid-Zeitschrift 101, 248-271.

Larsen, M.B., Boydston, A.J., 2014. Successive Mechanochemical Activation and Small Molecule Release in an Elastomeric Material. Journal of the American Chemical Society 136, 1276-1279.

Lee, C.K., Beiermann, B.A., Silberstein, M.N., Wang, J., Moore, J.S., Sottos, N.R., Braun, P.V., 2013. Exploiting Force Sensitive Spiropyrans as Molecular Level Probes. Macromolecules 46, 3746-3752.

Lee, C.K., Davis, D.A., White, S.R., Moore, J.S., Sottos, N.R., Braun, P.V., 2010. Force-induced redistribution of a chemical equilibrium. Journal of the American Chemical Society 132, 16107-16111. Lee, C.K., Diesendruck, C.E., Lu, E., Pickett, A.N., May, P.A., Moore, J.S., Braun, P.V., 2014. Solvent Swelling Activation of a Mechanophore in a Polymer Network. Macromolecules 47, 2690-2694. Lenhardt, J.M., Black, A.L., Beiermann, B.A., Steinberg, B.D., Rahman, F., Samborski, T., Elsakr, J., Moore, J.S., Sottos, N.R., Craig, S.L., 2011. Characterizing the mechanochemically active domains in gem-dihalocyclopropanated polybutadiene under compression and tension. Journal of Materials Chemistry 21, 8454-8459. 
Lenhardt, J.M., Ong, M.T., Choe, R., Evenhuis, C.R., Martinez, T.J., Craig, S.L., 2010. Trapping a diradical transition state by mechanochemical polymer extension. Science 329, 1057-1060.

Liang, J., Li, L., Niu, X., Yu, Z., Pei, Q., 2013. Elastomeric polymer light-emitting devices and displays. Nature Photonics 7, 817-824.

Lin, S., Cao, C., Wang, Q., Gonzalez, M., Dolbow, J.E., Zhao, X., 2014. Design of stiff, tough and stretchy hydrogel composites via nanoscale hybrid crosslinking and macroscale fiber reinforcement. Soft Matter 10, 7519-7527.

Mark, J.E., 1999. Improved elastomers through control of network chain-length distributions. Rubber chemistry and technology 72, 465-483.

Marko, J.F., Siggia, E.D., 1995. Stretching dna. Macromolecules 28, 8759-8770.

Maskarinec, S.A., Franck, C., Tirrell, D.A., Ravichandran, G., 2009. Quantifying cellular traction forces in three dimensions. Proceedings of the National Academy of Sciences 106, 22108-22113.

Morin, S.A., Shepherd, R.F., Kwok, S.W., Stokes, A.A., Nemiroski, A., Whitesides, G.M., 2012.

Camouflage and display for soft machines. Science 337, 828-832.

O’Bryan, G., Wong, B.M., McElhanon, J.R., 2010. Stress sensing in polycaprolactone films via an embedded photochromic compound. ACS applied materials \& interfaces 2, 1594-1600.

Ogden, R.W., Saccomandi, G., Sgura, I., 2006. On worm-like chain models within the three-dimensional continuum mechanics framework. Proceedings of the Royal Society A: Mathematical, Physical and Engineering Science 462, 749-768.

Park, S.-I., Xiong, Y., Kim, R.-H., Elvikis, P., Meitl, M., Kim, D.-H., Wu, J., Yoon, J., Yu, C.-J., Liu, Z., 2009. Printed assemblies of inorganic light-emitting diodes for deformable and semitransparent displays. Science 325, 977-981.

Peters, W., Ranson, W., 1982. Digital imaging techniques in experimental stress analysis. Optical Engineering 21, 213427-213427.

Ramirez, A.L.B., Kean, Z.S., Orlicki, J.A., Champhekar, M., Elsakr, S.M., Krause, W.E., Craig, S.L., 2013. Mechanochemical strengthening of a synthetic polymer in response to typically destructive shear forces. Nature Chemistry 5, 757-761.

Ribas-Arino, J., Marx, D., 2012. Covalent mechanochemistry: theoretical concepts and computational tools with applications to molecular nanomechanics. Chemical reviews 112, 5412-5487.

Rubinstein, M., Colby, R., 2003. Polymers Physics. Oxford.

Sagara, Y., Kato, T., 2009. Mechanically induced luminescence changes in molecular assemblies. Nature Chemistry 1, 605-610.

Sekitani, T., Nakajima, H., Maeda, H., Fukushima, T., Aida, T., Hata, K., Someya, T., 2009. Stretchable active-matrix organic light-emitting diode display using printable elastic conductors. Nature materials 8 , 494-499.

Shepherd, R.F., Ilievski, F., Choi, W., Morin, S.A., Stokes, A.A., Mazzeo, A.D., Chen, X., Wang, M., Whitesides, G.M., 2011. Multigait soft robot. Proceedings of the National Academy of Sciences 108, 20400-20403.

Silberstein, M.N., Cremar, L.D., Beiermann, B.A., Kramer, S.B., Martinez, T.J., White, S.R., Sottos, N.R., 2014. Modeling mechanophore activation within a viscous rubbery network. Journal of the Mechanics and Physics of Solids 63, 141-153.

Silberstein, M.N., Min, K., Cremar, L.D., Degen, C.M., Martinez, T.J., Aluru, N.R., White, S.R., Sottos, N.R., 2013. Modeling mechanophore activation within a crosslinked glassy matrix. Journal of Applied Physics 114, 023504.

Sun, J.-Y., Zhao, X., Illeperuma, W.R., Chaudhuri, O., Oh, K.H., Mooney, D.J., Vlassak, J.J., Suo, Z., 2012. Highly stretchable and tough hydrogels. Nature 489, 133-136.

Treloar, L., Riding, G., 1979. A non-Gaussian theory for rubber in biaxial strain. I. Mechanical properties. Proceedings of the Royal Society of London. A. Mathematical and Physical Sciences 369, 261-280.

Treloar, L.R.G., 1975. The physics of rubber elasticity. Oxford University Press. 
Wang, J., Kouznetsova, T.B., Kean, Z.S., Fan, L., Mar, B.D., Martínez, T.J., Craig, S.L., 2014a. A Remote Stereochemical Lever Arm Effect in Polymer Mechanochemistry. Journal of the American Chemical Society 136, 15162-15165.

Wang, Q., Gossweiler, G.R., Craig, S.L., Zhao, X., 2014b. Cephalopod-inspired design of electromechano-chemically responsive elastomers for on-demand fluorescent patterning. Nature

Communications 5, 4899.

White, M.S., Kaltenbrunner, M., Głowacki, E.D., Gutnichenko, K., Kettlgruber, G., Graz, I., Aazou, S., Ulbricht, C., Egbe, D.A., Miron, M.C., 2013. Ultrathin, highly flexible and stretchable PLEDs. Nature Photonics 7, 811-816.

Wu, P., Van der Giessen, E., 1992. On improved 3-D non-Gaussian network models for rubber elasticity. Mechanics research communications 19, 427-433.

Xu, Y., Engl, W.C., Jerison, E.R., Wallenstein, K.J., Hyland, C., Wilen, L.A., Dufresne, E.R., 2010.

Imaging in-plane and normal stresses near an interface crack using traction force microscopy.

Proceedings of the National Academy of Sciences 107, 14964-14967.

Yeo, J.-S., Emery, T., Combs, G., Korthuis, V., Mabeck, J., Hoffman, R., Koch, T., Zhou, Z.-L., Henze, D., 2010. 69.4: Novel Flexible Reflective Color Media Integrated with Transparent Oxide TFT Backplane. SID Symposium Digest of Technical Papers 41, 1041-1044.

Yu, Z., Niu, X., Liu, Z., Pei, Q., 2011. Intrinsically Stretchable Polymer Light-Emitting Devices Using Carbon Nanotube-Polymer Composite Electrodes. Advanced Materials 23, 3989-3994.

Zhang, H., Chen, Y., Lin, Y., Fang, X., Xu, Y., Ruan, Y., Weng, W., 2014. Spiropyran as a

Mechanochromic Probe in Dual Cross-Linked Elastomers. Macromolecules 47, 6783-6790.

Zhao, X., 2012. A theory for large deformation and damage of interpenetrating polymer networks. Journal of the Mechanics and Physics of Solids 60, 319-332.

Zhao, X., 2014. Multi-scale multi-mechanism design of tough hydrogels: building dissipation into stretchy networks. Soft Matter 10, 672-687. 


\section{Figures and Figure Captions}

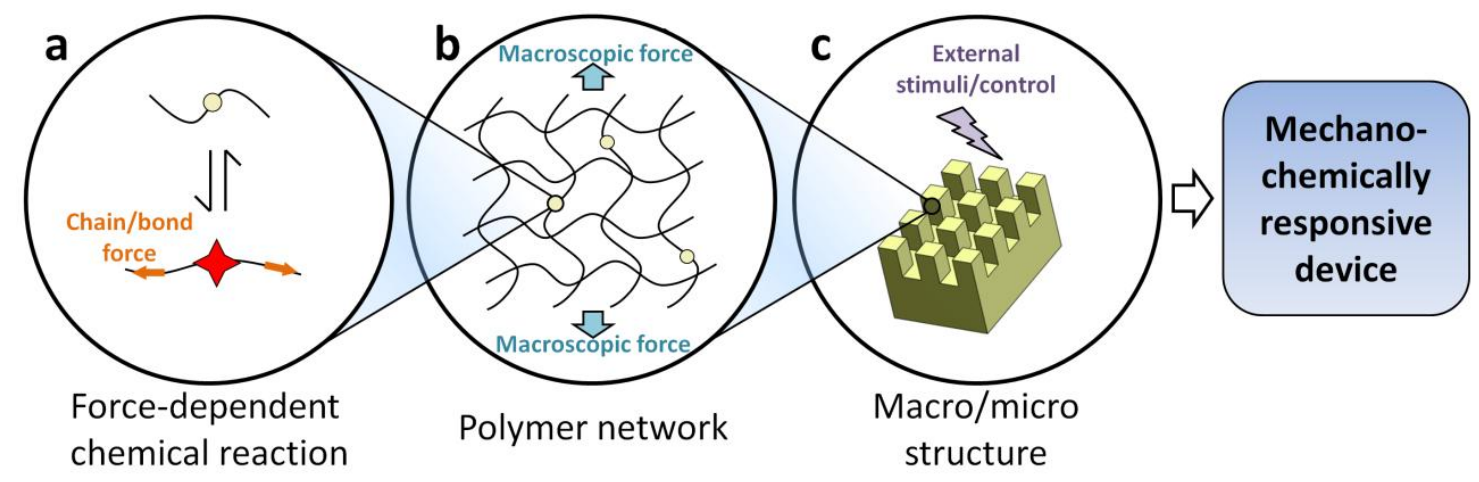

Fig. 1. Three levels of designs for the mechanochemically responsive devices. (a) On the molecular level, the development and optimization of new mechanophores capable of force-dependent chemical reactions. (b) On the network level, designing the architectures of polymer networks, on which mechanophores are coupled. (c) On the device level, designing structures and actuation mechanisms of the devices. 

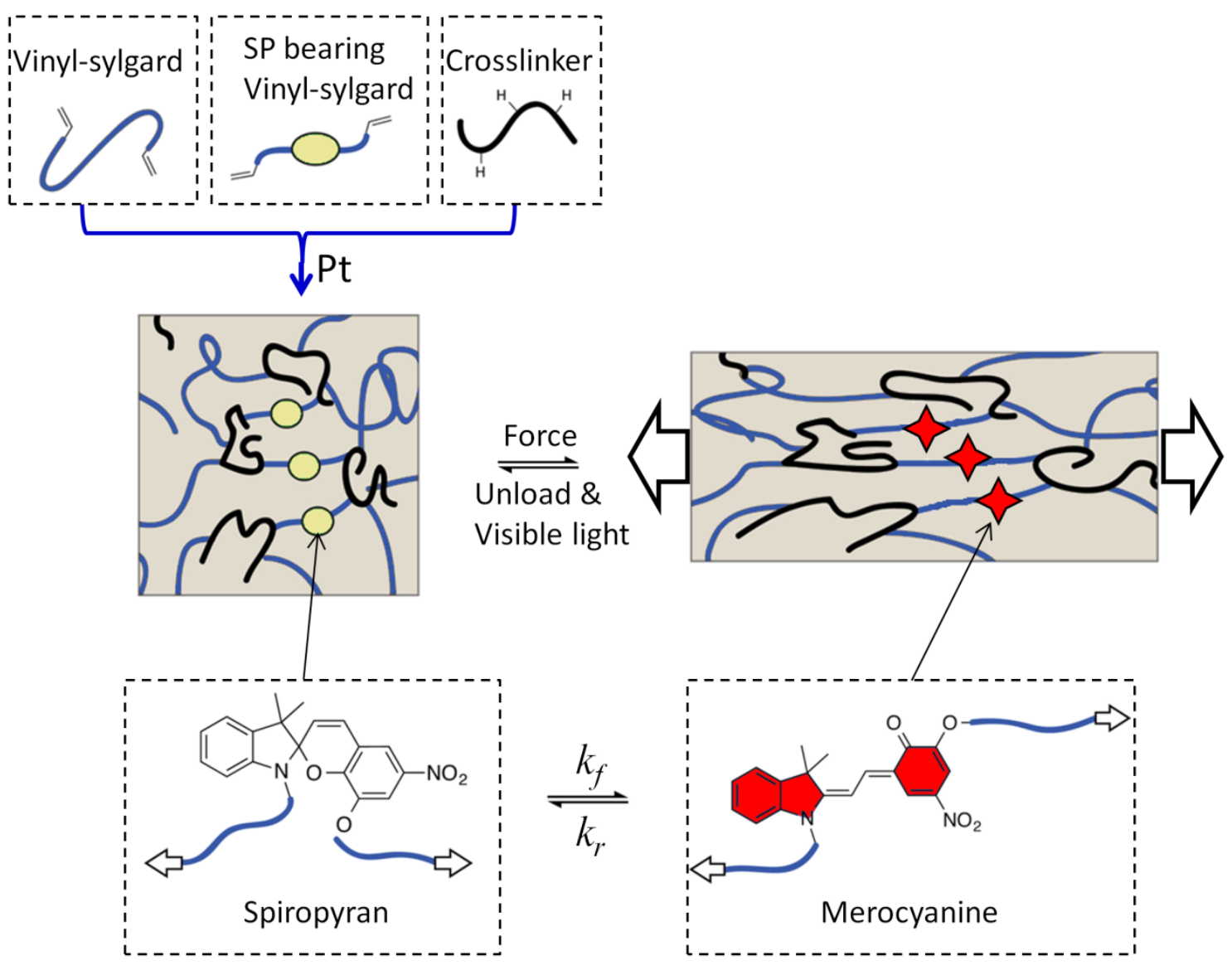

Fig. 2. Schematics for covalently incorporating spiropyrans into the Sylgard network and activation of mechanophores. The MCR elastomer under sufficiently large deformation induces significantly high forces in polymer chains, which triggers a $6-\pi$ electrocyclic ring-opening reaction that transit spiropyran into merocynanine. 
a

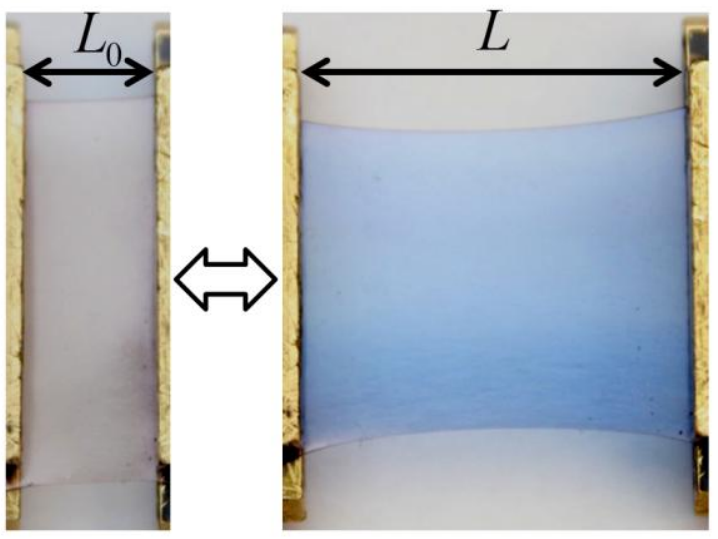

b

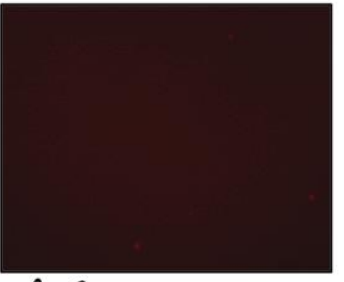

$\lambda=1$

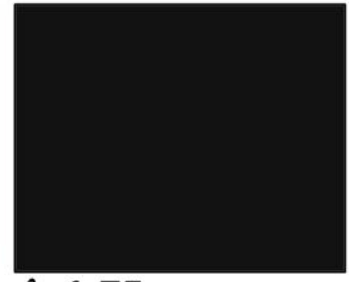

$\lambda=1.75$
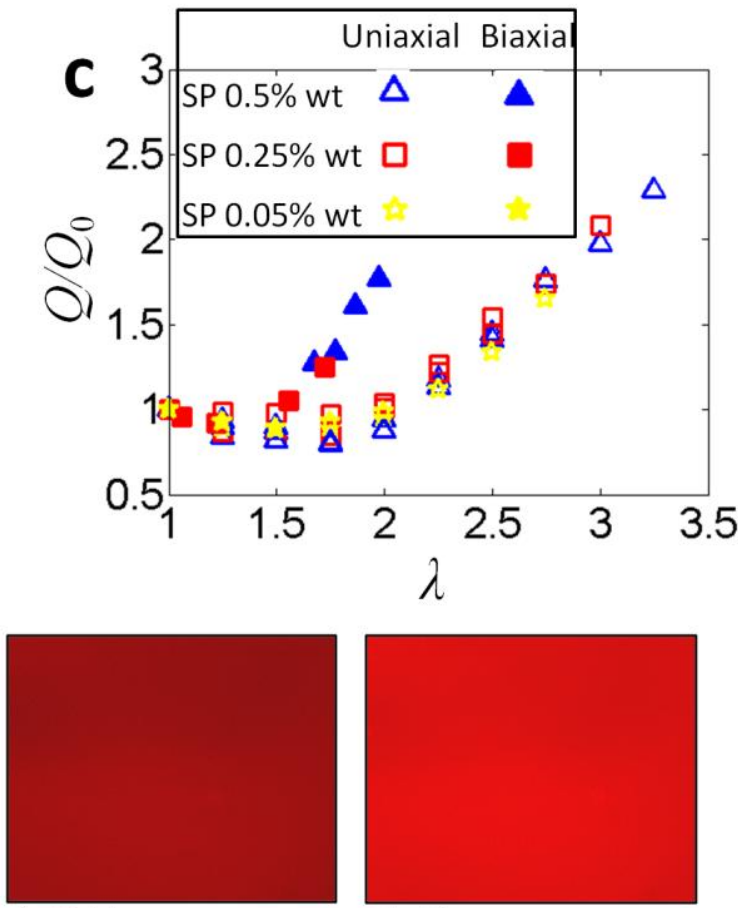

$\lambda=2.5$

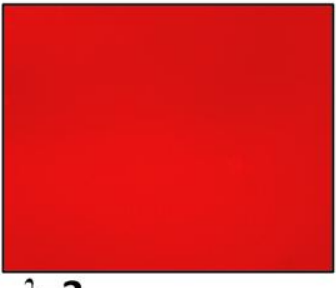

$\lambda=3$

Fig. 3. (a) An MCR elastomer stretched under a pure-shear condition changes the color from the initially pale yellow to dark blue (Wang et al., 2014b). (b) Fluorescence images of the elastomer film under different uniaxial stretches. (c) The normalized fluorescence intensity plotted as a function of the applied stretch of the uniaxial and equibiaxial tests. The data in this figure are taken from Wang et al., 2014b. 


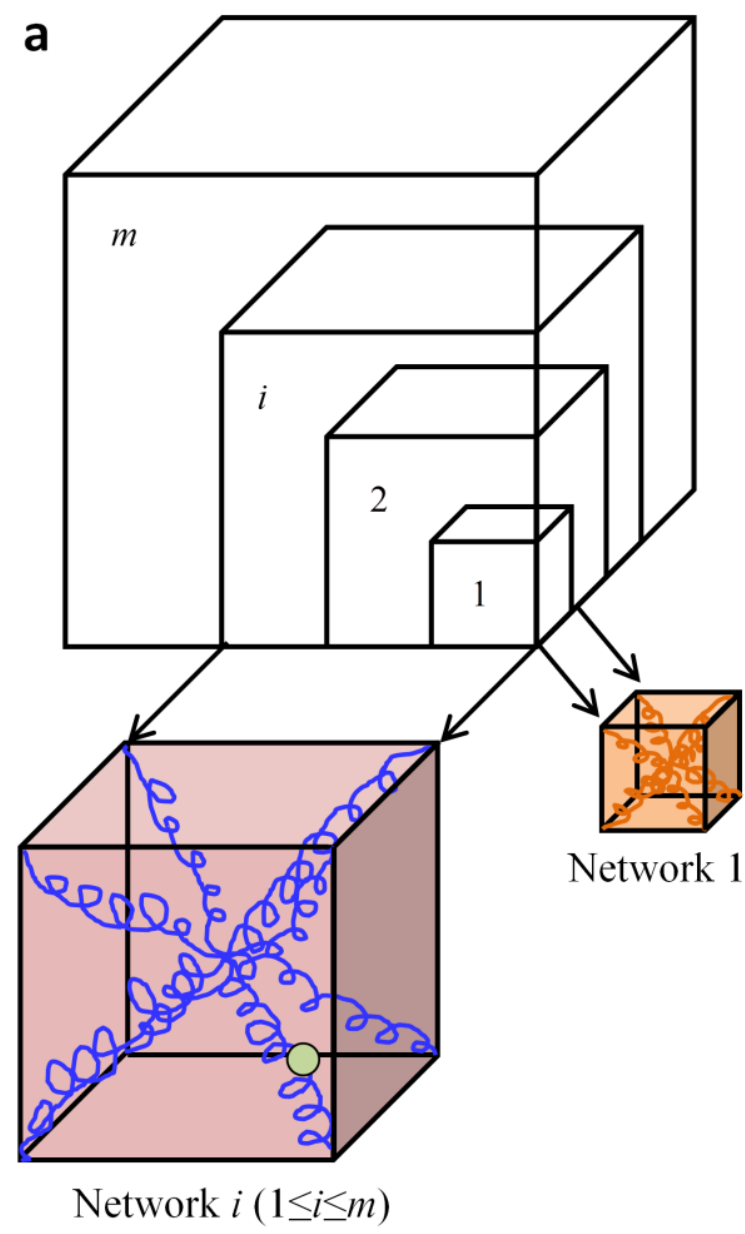

b
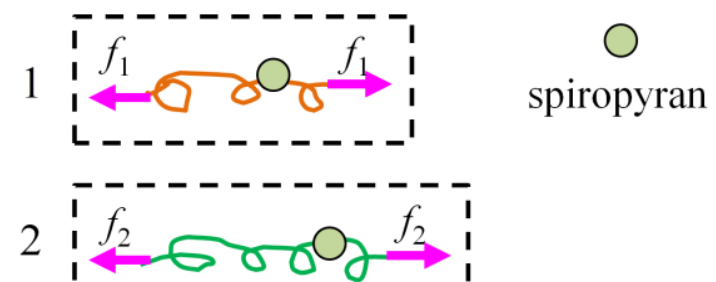

I - - - - - - - - - I<smiles>C1CCCC1</smiles>
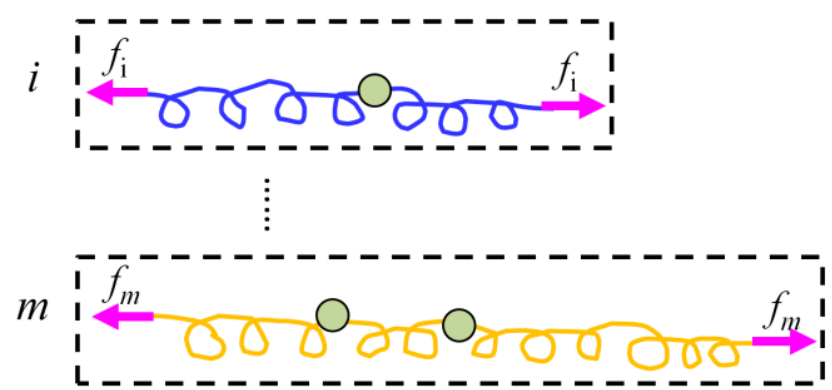

Fig. 4. (a) The MCR elastomer consists of $m$ types of polymer networks interpenetrated with one another.

The link number of polymer chains (i.e., chain length) is identical within the same type of network. (b) Under the identical deformations, the forces applied to mechanophores bound to polymer chains of different lengths are different. 


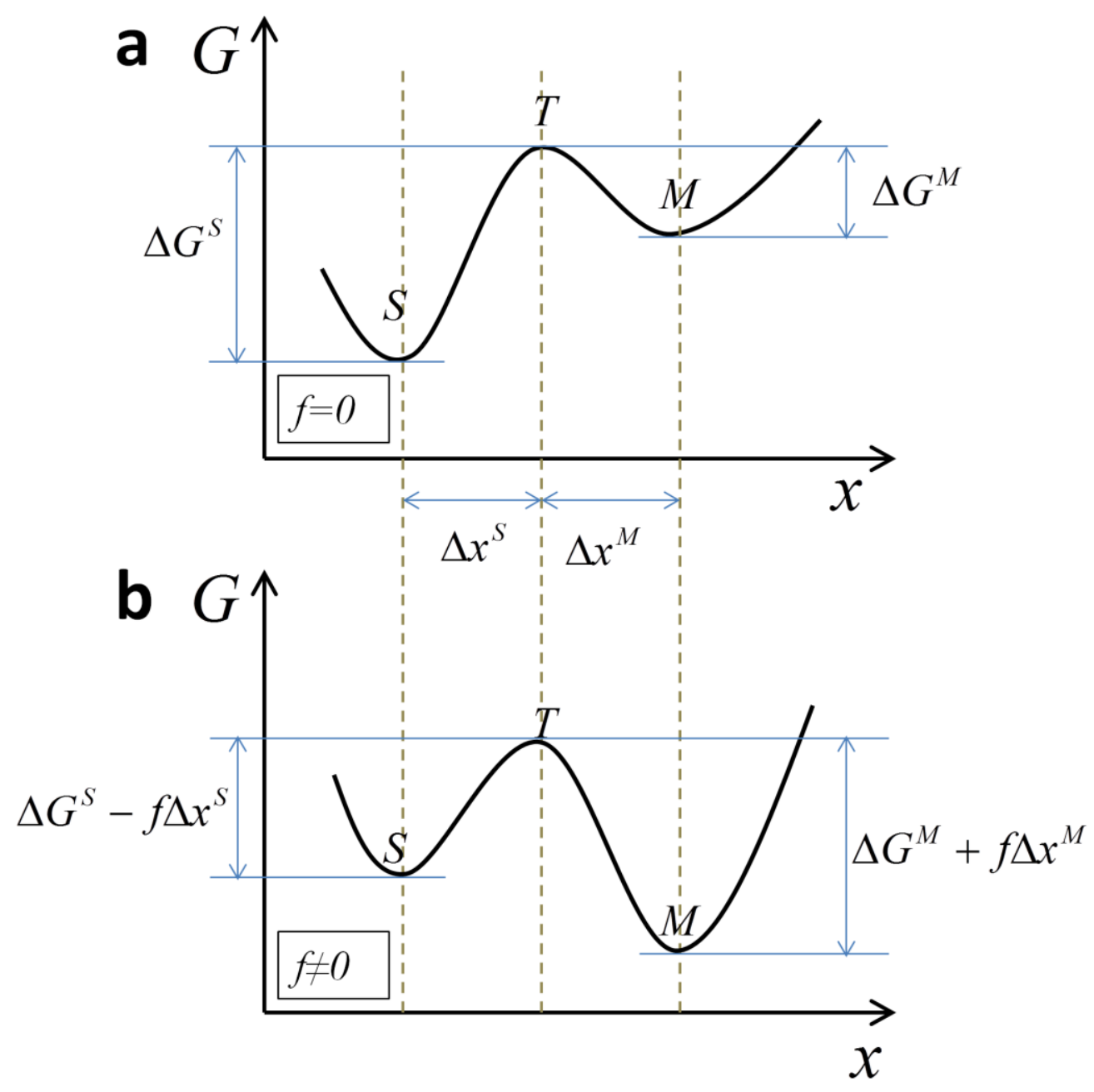

Fig. 5. Potential energy landscape of the mechanophores reactions under (a) undeformed and (b) deformed states. "S" stands for spiropyran, "M" stands for merocyanines, and "T" stands for the transition state. 

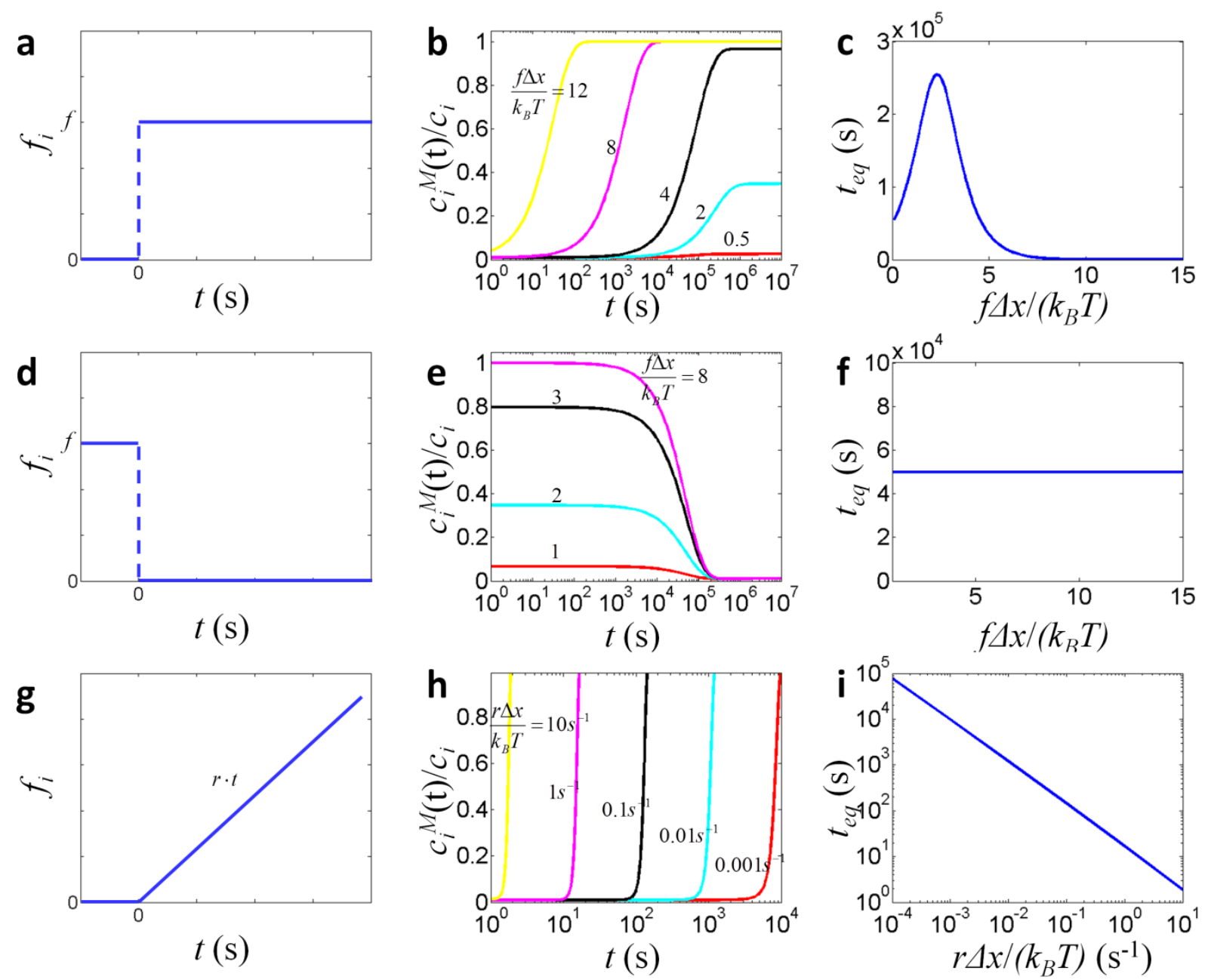

Fig. 6. Different profiles of time-dependent chain forces (a, c, g) give different transient evolutions of merocyanine concentrations $(\mathrm{b}, \mathrm{e}, \mathrm{h})$ and different time scales for the chemical reaction to reach equilibrium states (c, f ,i). The zero-force forward and reverse reaction rates are set to be $k^{f 0}=1.94 \times 10^{-7} s^{-1}$ and $k^{r 0}=1.99 \times 10^{-5} s^{-1}$, respectively. 

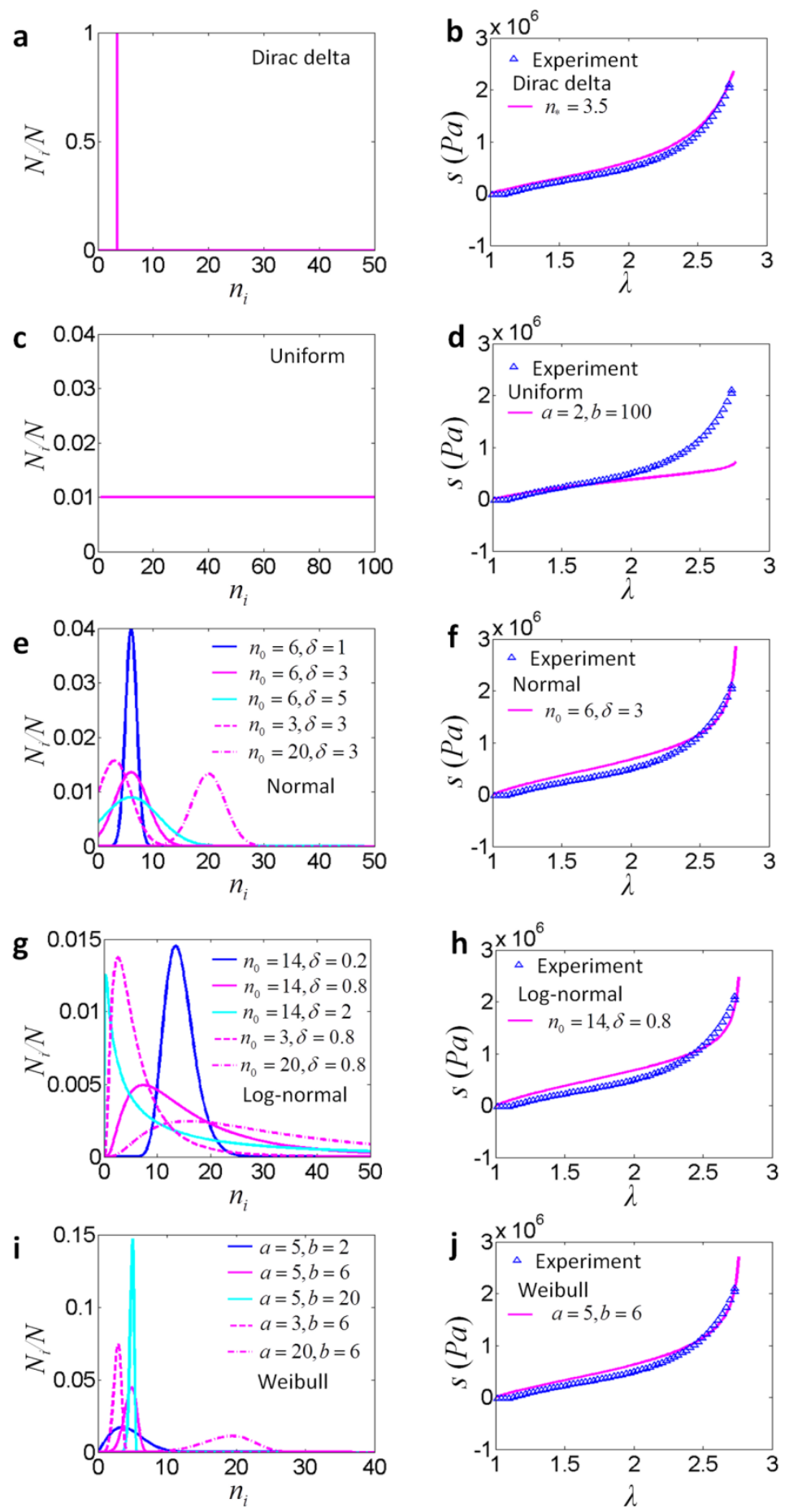

Fig. 7. Chain-length distributions (left) and stress-stretch curves (right) for the (a, b) Dirac delta distribution, (c, d) uniform distribution, (e, f) normal distribution, $(\mathrm{g}, \mathrm{h})$ log-normal distribution, and (i, j) Weibull distribution, respectively. The parameters in the right column are chosen to give the best fit to the experimental data. 

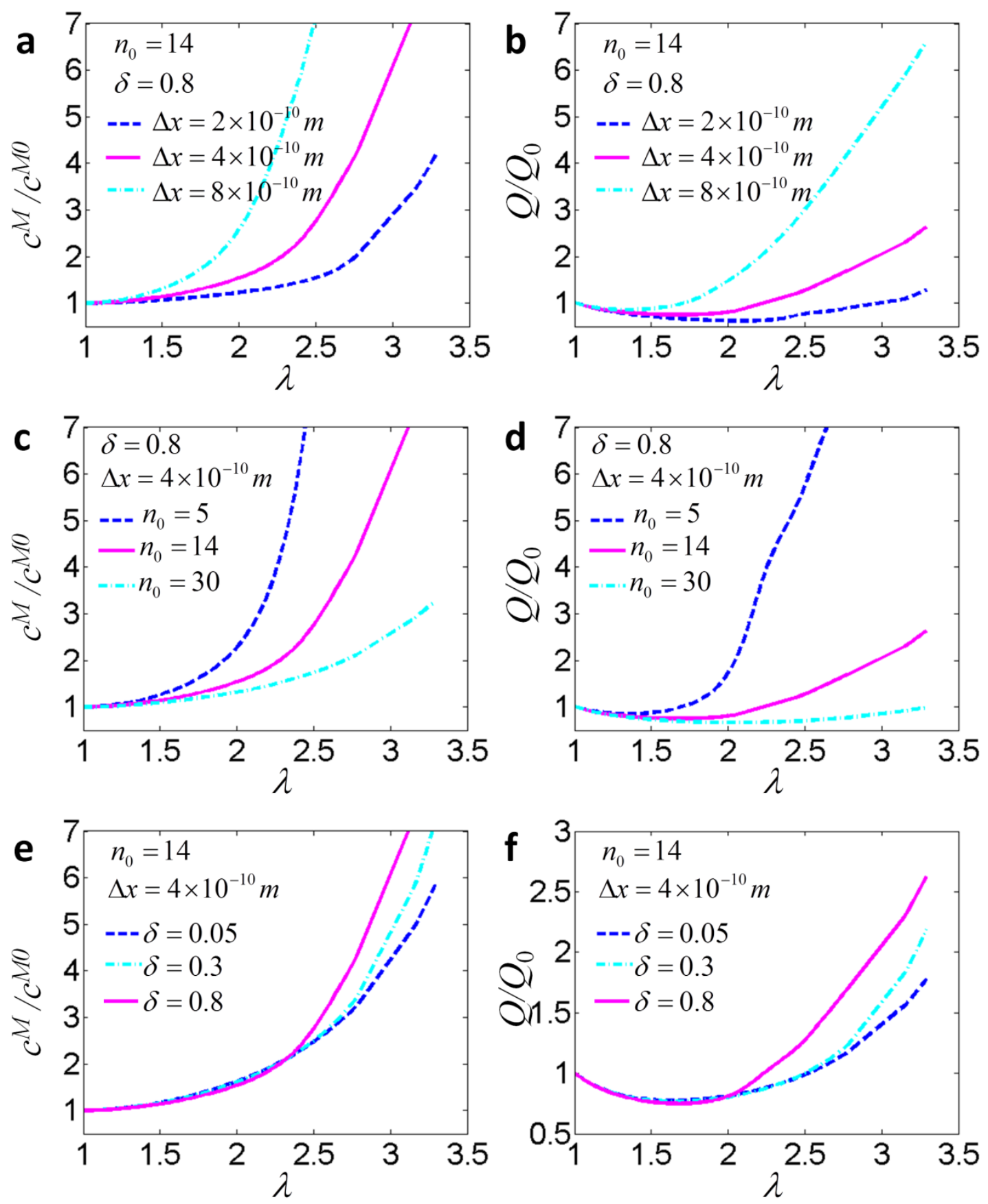

Fig. 8. Effects of (a, b) effective reaction distance $\Delta x$, (c, d) the mean $\ln n_{0}$ and (e, f) standard deviation $\delta$ of the logarithm of the chain-link-number $\ln n_{i}$, on the predicted concentration of merocyanine and resulting fluorescence ratios, respectively. The adopted chain-length distribution is the log-normal distribution. 

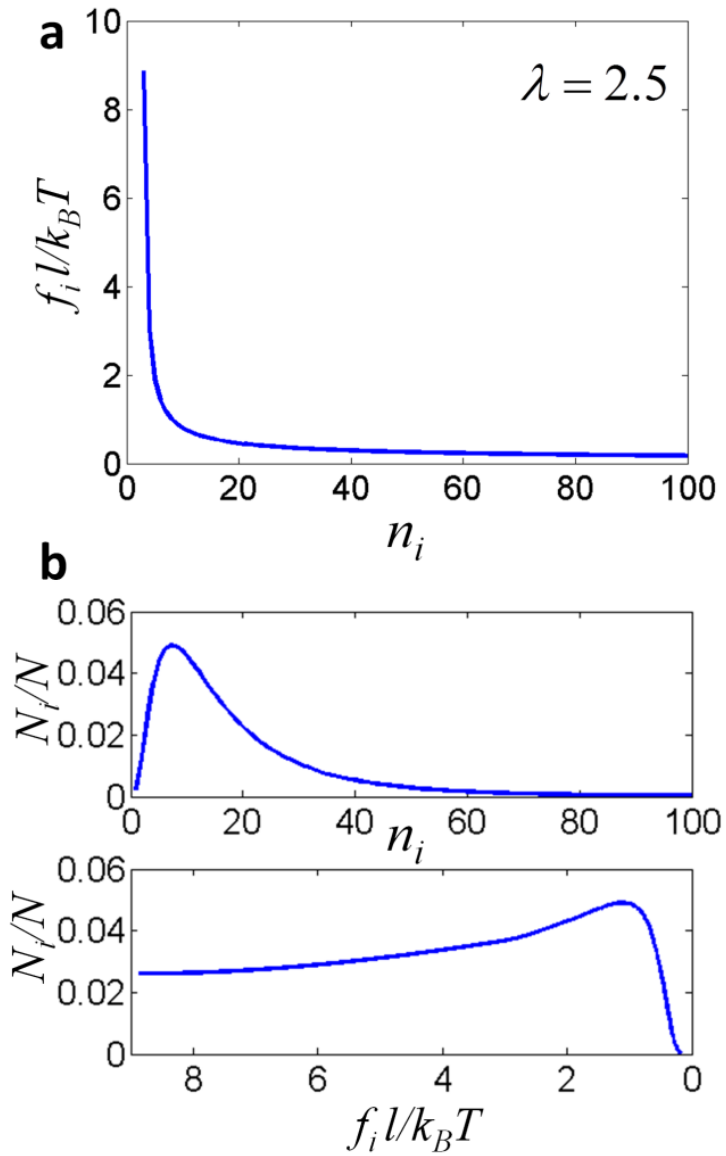

Fig. 9. (a) The chain force plotted as a function of the link number. (2) A chain-length distribution and corresponding chain-force distribution. The chain length distribution follows the log-normal distribution with $N=8 \times 10^{25}, \ln n_{0}=\ln 14$ and $\delta=0.8$. The MCR elastomer is under uniaxial pure-shear tension with the uniaxial stretch $\lambda=2.5$. 

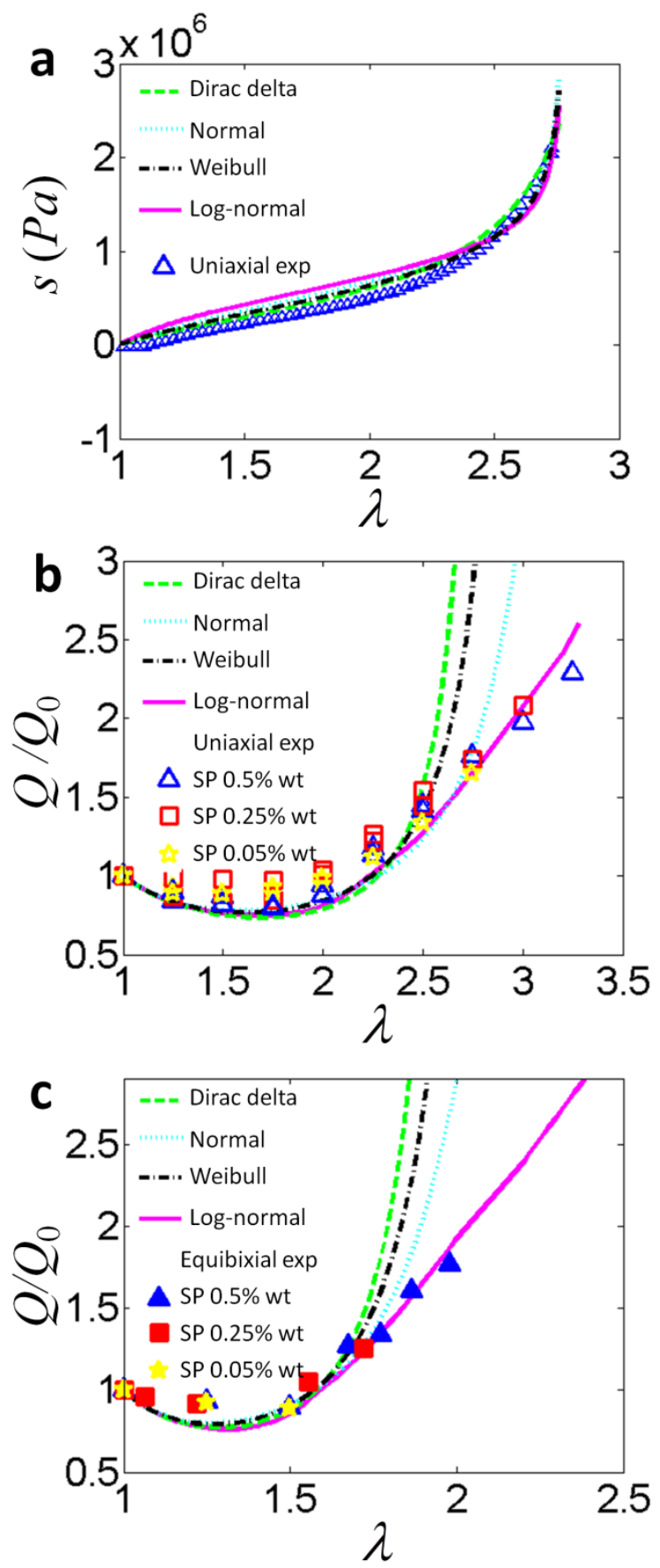

Fig. 10. (a) Theoretically predicted and experimentally measured stress-stretch curves of the uniaxial tension tests (replots of Figs. 7b, 7f, $7 \mathrm{~h}$ and $7 \mathrm{j}$ ). Comparison between the theoretically predicted and experimentally observed fluorescence ratios under (b) the uniaxial pure-shear tension and (c) the equibiaxial tension. The model with a Dirac delta distribution adopts $n_{*}=3.5$ and $\Delta x=8 \times 10^{-11} \mathrm{~m}$. The 
model with a normal distribution adopts $n_{0}=6, \delta=3$ and $\Delta x=2.5 \times 10^{-10} \mathrm{~m}$. The model with a lognormal distribution adopts $\ln n_{0}=\ln 14, \delta=0.8$ and $\Delta x=4 \times 10^{-10} \mathrm{~m}$. The model with a Weibull distribution adopts $a=5, b=6$ and $\Delta x=1.5 \times 10^{-10} \mathrm{~m}$. 

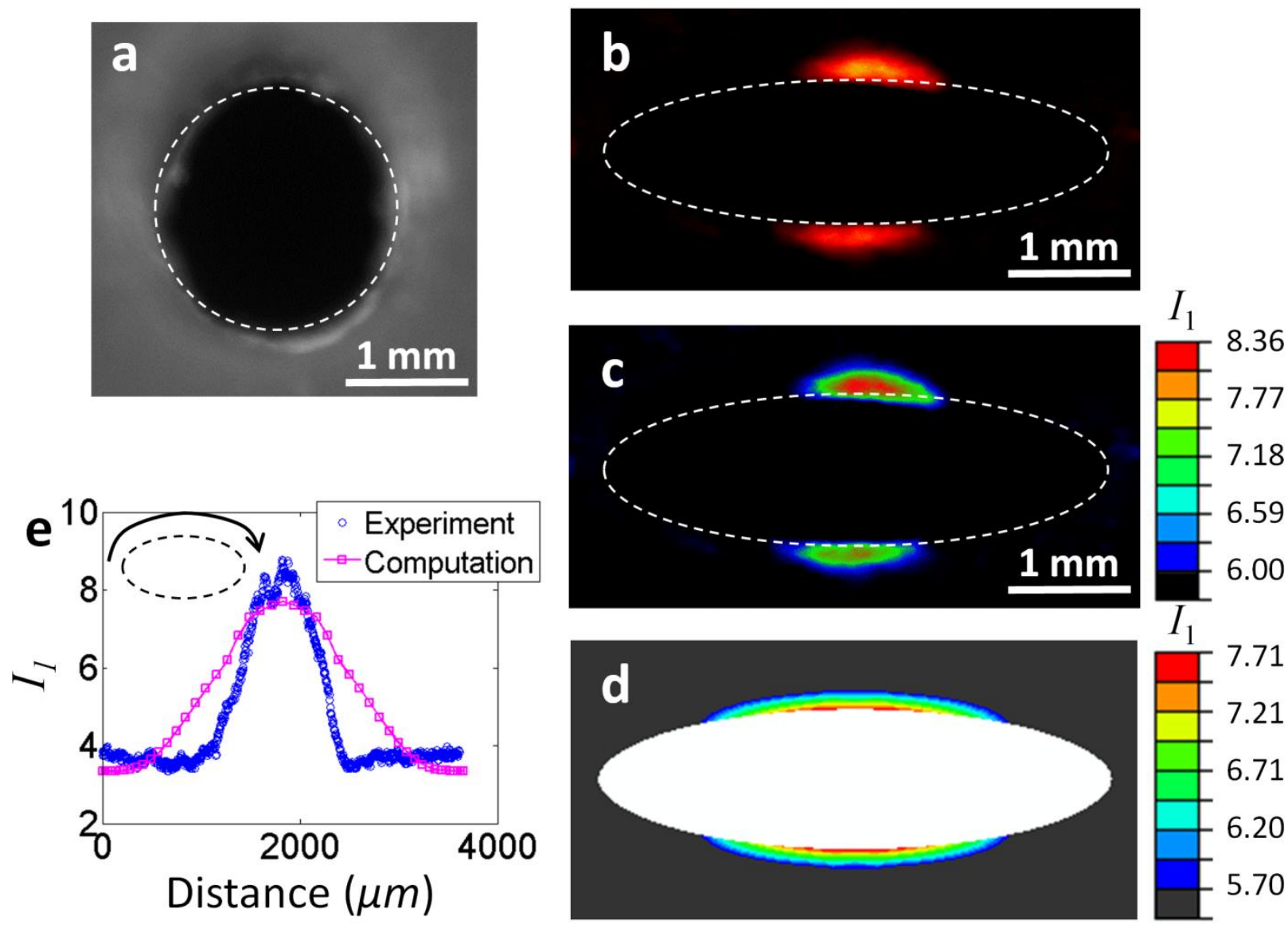

Fig. 11. (a) Optical image of a circular hole in an MCR elastomer film at undeformed state. (b) Observed fluorescence, (c) converted first invariants from the fluorescence and (d) calculated first invariants from the finite-element model of regions around the hole, which is deformed into an ellipse under uniaxial stretch of the MCR elastomer. (e) Comparison of the observed first invariants from fluorescence and calculated first invariants from the finite-element model along the boundary of the elliptical hole. 

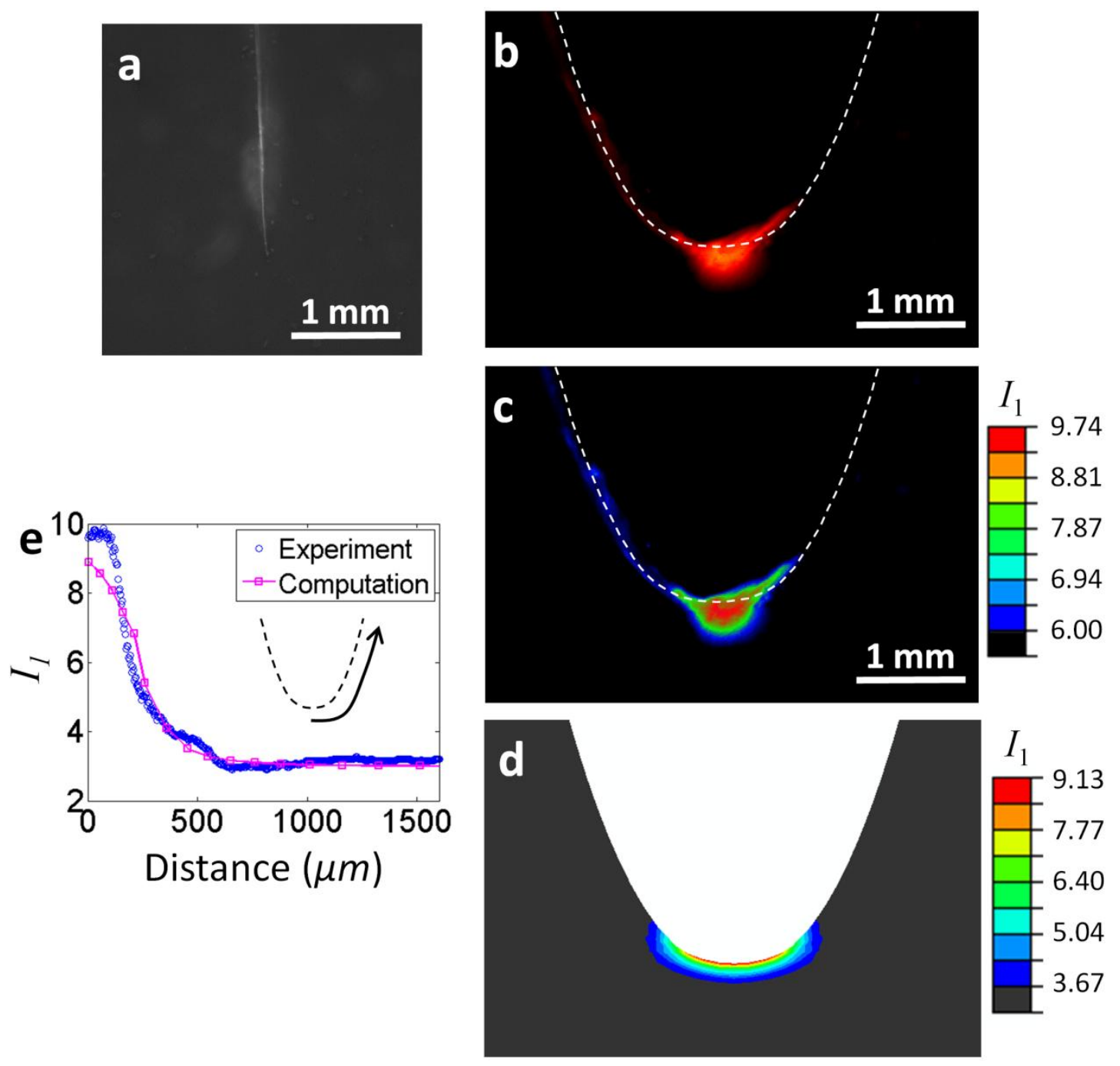

Fig. 12. (a) Optical image of a crack in an MCR elastomer film at undeformed state. (b) Observed fluorescence, (c) converted first invariants from the fluorescence and (d) calculated first invariants from the finite-element model of regions around the crack tip, which is blunted due to the uniaxial stretch of the MCR elastomer. (e) Comparison of the observed first invariants from fluorescence and calculated first invariants from the finite-element model along the blunted crack. 

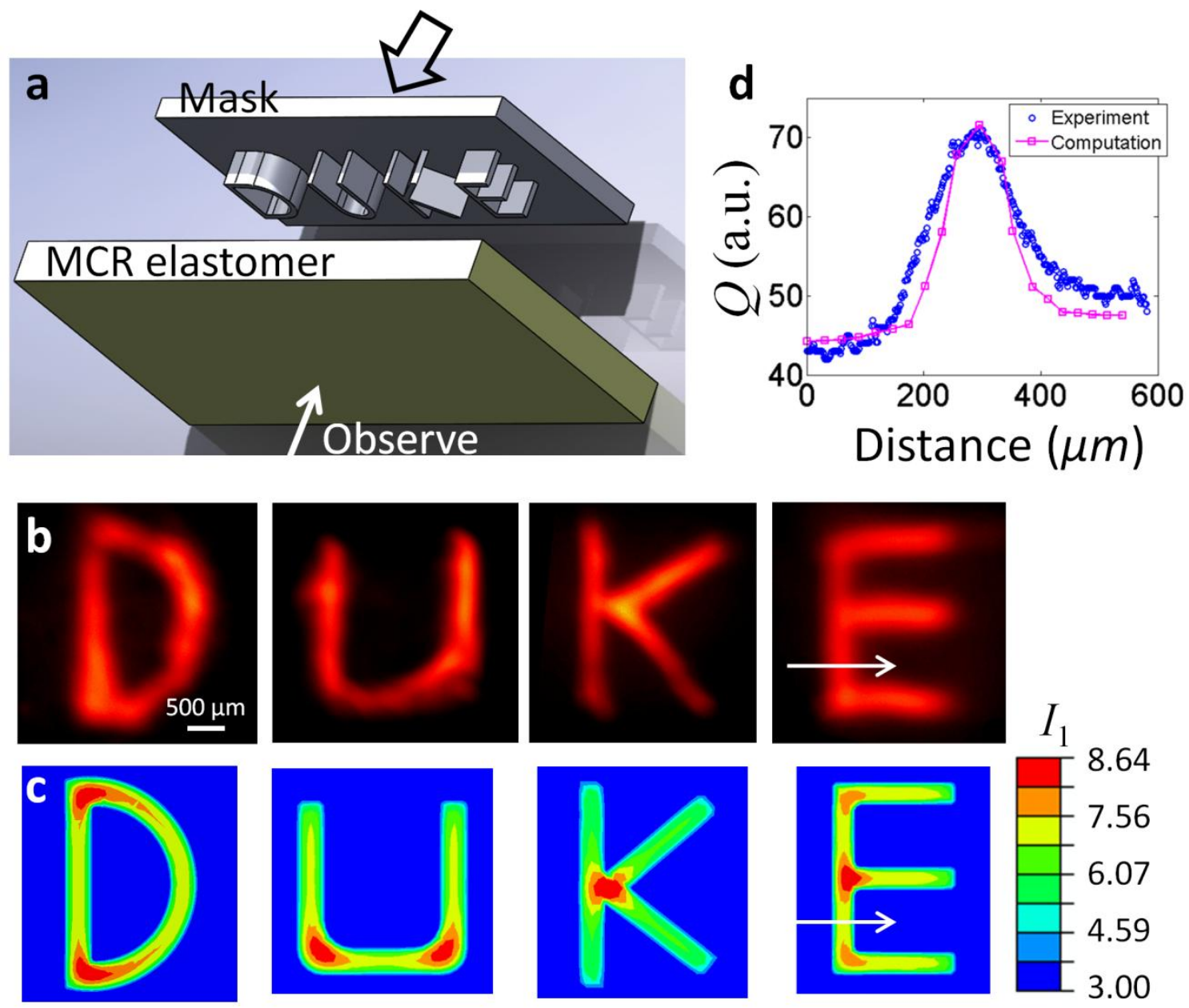

Fig. 13. (a) Schematic of imprinting letters "DUKE" on an MCR elastomer. (b) Fluorescence image and (c) the first invariant mapping of the deformed MCR elastomer. (d) The observed and simulated fluorescence intensity along the indicated path across the letter "E". 

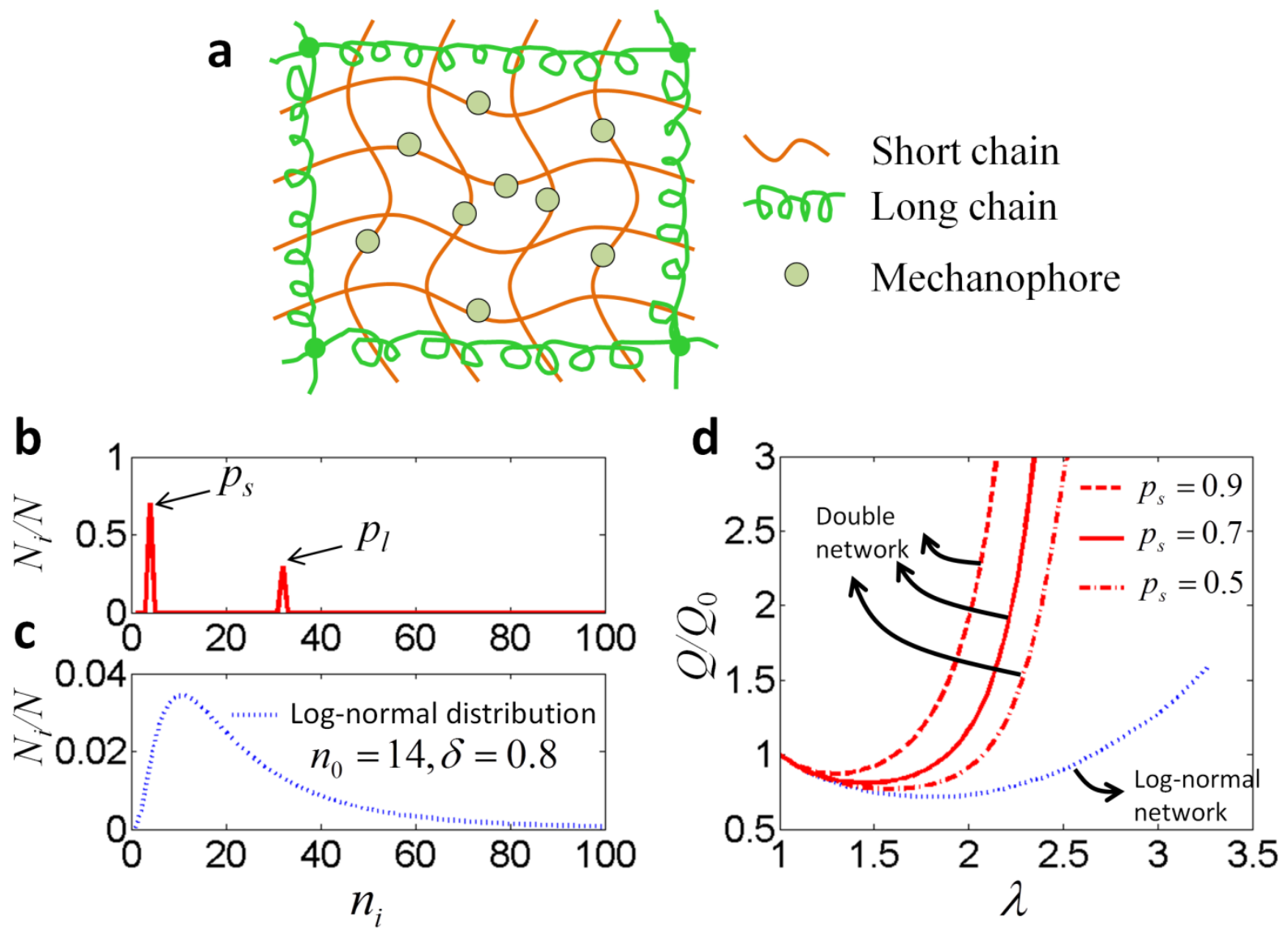

Fig. 14. (a) Schematics of a double network MCR elastomer, wherein mechanophores are selectively attached to the network with short chains. (b) The chain-length distribution of a double-network MCR elastomer, where $p_{s}$ and $p_{l}=1-p_{s}$ denote the probability densities of the short and long chains, respectively. In the double-network elastomer, mechanophores are only coupled to the short-chain network. (c) The log-normal chain-length distribution of an MCR elastomer. In the log-normal-network elastomer, mechanophores are homogeneously distributed in all networks. (d) Predicted fluorescence ratios for double-network MCR elastomers and the MCR elastomer with log-normal chain-length distribution. 